\title{
A CONVENIENT NOTION OF COMPACT SET FOR GENERALIZED FUNCTIONS
}

\author{
PAOLO GIORDANO AND MICHAEL KUNZINGER
}

\begin{abstract}
We introduce the notion of functionally compact sets into the theory of nonlinear generalized functions in the sense of Colombeau. The motivation behind our construction is to transfer, as far as possible, properties enjoyed by standard smooth functions on compact sets into the framework of generalized functions. Based on this concept, we introduce spaces of compactly supported generalized smooth functions that are close analogues to the test function spaces of distribution theory. We then develop the topological and functional analytic foundations of these spaces.
\end{abstract}

\section{INTRODUCTION}

A main advantage of nonlinear generalized functions in the sense of Colombeau as compared to Schwartz distributions is the fact that they can be viewed as settheoretic maps on domains consisting of generalized points. This change of perspective allows to develop several branches of the theory in close analogy to classical analysis, and thereby has become increasingly important in recent years (cf., e.g., $[26,4,2,11,12,27,3,16,6,18])$. In particular, appropriate topologies on spaces of nonlinear generalized functions, the so called sharp topologies (see below for the definition), have been introduced in $[28,29]$ and have since been studied by many authors. Apart from their central position in the structure theory of Colombeau algebras, they also supply the foundation for applications in the theory of nonlinear partial differential equations (e.g., for a suitable concept of well-posedness).

From the point of view of analysis, a key notion underlying many existence results is that of compactness. It turns out, however, that sharply compact subsets of generalized points display certain unwanted properties: e.g., no infinite subset of $\mathbb{R}^{n}$ is sharply compact since the trace of the sharp topology on subsets of $\mathbb{R}^{n}$ is

2010 Mathematics Subject Classification. 46F30,46A13,13J99.

Key words and phrases. Functionally compact sets, Colombeau generalized functions, generalized smooth functions, locally convex modules.

P. Giordano has been supported by grant P25116 and P25311 of the Austrian Science Fund FWF. M. Kunzinger has been supported by grants P23714 and P25326 of the Austrian Science Fund FWF. 
discrete. In fact, this is a necessary consequence of the set $\widetilde{\mathbb{R}}$ of generalized numbers containing actual infinitesimals, hence seems unavoidable also in alternative approaches, cf. [17, Prop. 2.1] and [18, Thm. 25].

The importance of a convenient notion of compactness for nonlinear generalized functions has been recognized by several authors, most recently in [1]. The approach we take in the present paper is to introduce an appropriate concept of compactly supported generalized function, and then to study spaces consisting of such functions, which, in analogy to the test function space $\mathcal{D}(\Omega)$ in distribution theory, we denote by $\mathcal{G D}(U)$. The domain $U$ here is a set of generalized points. Based on Garetto's theory of locally convex $\widetilde{\mathbb{C}}$-modules $[11,12,14]$ we then develop the topological and functional analytic foundations of these spaces. We find that they are indeed close analogues of the classical spaces of test functions in that they are countable strict inductive limits of complete metric spaces $\mathcal{G} \mathcal{D}_{K}(U)$ (analogues of $\mathcal{D}_{K}(\Omega)$ in distribution theory) satisfying properties paralleling those of the classical strict (LF)-spaces $\mathcal{D}(\Omega)$.

The plan of the paper is as follows: In the remainder of this introduction we fix some basic notions used throughout this work. Section 2 introduces what we call functionally compact sets, based on work by Oberguggenberger and Vernaeve in [27]. Building on this, in Section 3 we define compactly supported generalized smooth functions (GSF), as well as the corresponding spaces $\mathcal{G D}(U)$ and $\mathcal{G D}_{K}(U)$. We also show that every Colombeau generalized function $f \in \mathcal{G}^{s}(\Omega)$ (in particular, every Schwartz distribution) defines a compactly supported GSF $\bar{f}: \widetilde{\mathbb{R}} \longrightarrow \widetilde{\mathbb{R}}$ that coincides with $f$ on $\widetilde{\Omega}_{c}$. In order to obtain appropriate topologies on these spaces, we define so-called generalized norms in Section 4. These are maps that share the basic properties of classical norms, yet take values in $\widetilde{\mathbb{R}}$, thereby generalizing a standard alternative description of the sharp topology on generalized numbers (cf. $[4,17])$. In Sections 5 and 6 these generalized norms are employed to endow the spaces $\mathcal{G D}_{K}(U)$ with metric topologies. In particular, in Section 5.1 we study connections between non-Archimedean properties and Hausdorff topological vector spaces of generalized functions, proving an impossibility theorem: there does not exist a Hausdorff topological vector subspace of the Colombeau special algebra which contains the Dirac delta and even a single trace of an open set of the sharp topology. The completeness of the spaces $\mathcal{G} \mathcal{D}_{K}(U)$ is established in Section 7. In the final Section 8 we derive the fundamental functional analytic properties of the space $\mathcal{G} \mathcal{D}(U)$.

1.1. Basic notions. Our main references for Colombeau's theory are [8, 9, 25, 22]. The special Colombeau algebra $\mathcal{G}^{s}(\Omega)$ over an open subset $\Omega$ of $\mathbb{R}^{n}$ is defined as the quotient $\mathcal{E}_{M}^{s}(\Omega) / \mathcal{N}^{s}(\Omega)$, where (setting $I:=(0,1]$ and noting that in the naturals 
$\mathbb{N}=\{0,1,2,3 \ldots\}$ we include zero.)

$$
\begin{aligned}
& \mathcal{E}_{M}^{s}(\Omega):=\left\{\left(u_{\varepsilon}\right) \in \mathcal{C}^{\infty}(\Omega)^{I}\left|\forall K \Subset \Omega \forall \alpha \in \mathbb{N}^{n} \exists N \in \mathbb{N}: \sup _{x \in K}\right| \partial^{\alpha} u_{\varepsilon}(x) \mid=O\left(\varepsilon^{-N}\right)\right\} \\
& \mathcal{N}^{s}(\Omega):=\left\{\left(u_{\varepsilon}\right) \in \mathcal{C}^{\infty}(\Omega)^{I}\left|\forall K \Subset \Omega \forall \alpha \in \mathbb{N}^{n} \forall m \in \mathbb{N}: \sup _{x \in K}\right| \partial^{\alpha} u_{\varepsilon}(x) \mid=O\left(\varepsilon^{m}\right)\right\} .
\end{aligned}
$$

Elements of $\mathcal{E}_{M}^{s}(\Omega)$ are called moderate, those of $\mathcal{N}^{s}(\Omega)$ are called negligible. Nets in $\mathcal{E}_{M}^{s}(\Omega)$ are written as $\left(u_{\varepsilon}\right)$, and $u=\left[u_{\varepsilon}\right]$ denotes the corresponding equivalence class in $\mathcal{G}^{s}(\Omega)$. For $\left(u_{\varepsilon}\right) \in \mathcal{N}^{s}(\Omega)$ we also write $\left(u_{\varepsilon}\right) \sim 0$. We will abbreviate 'Colombeau generalized function' by CGF. $\mathcal{G}^{s}(-)$ is a fine sheaf of differential algebras and there exist sheaf embeddings (based on smoothing via convolution) of the space of Schwartz distributions $\mathcal{D}^{\prime}$ into $\mathcal{G}^{s}$ (cf. [22]).

Given $\Omega \subseteq \mathbb{R}^{n}$ open, the space of generalized points in $\Omega$ is $\widetilde{\Omega}=\Omega_{M} / \sim$, where $\Omega_{M}=\left\{\left(x_{\varepsilon}\right) \in \Omega^{I}|\exists N \in \mathbb{N}:| x_{\varepsilon} \mid=O\left(\varepsilon^{-N}\right)\right\}$ is called the set of moderate nets and $\left(x_{\varepsilon}\right) \sim\left(y_{\varepsilon}\right)$ if $\left|x_{\varepsilon}-y_{\varepsilon}\right|=O\left(\varepsilon^{m}\right)$ for every $m \in \mathbb{N}$. In the particular case $\Omega=\mathbb{R}$ we obtain the ring of Colombeau generalized numbers (CGN) $\widetilde{\mathbb{R}}=\mathbb{R}_{M} / \sim$ (and analogously for $\widetilde{\mathbb{C}}$ ), which can also be written as $\widetilde{\mathbb{R}}=\mathbb{R}_{M} / \mathcal{N}^{s}$, where $\mathcal{N}^{s}$ is the set of all negligible nets of real numbers $\left(x_{\varepsilon}\right) \in \mathbb{R}^{I}$, i.e. such that $\left(x_{\varepsilon}\right) \sim 0$. $\widetilde{\mathbb{R}}$ is an ordered ring with respect to its natural order relation: $x \leq y$ iff there are representatives $\left(x_{\varepsilon}\right)$ and $\left(y_{\varepsilon}\right)$ such that $x_{\varepsilon} \leq y_{\varepsilon}$ for $\varepsilon$ sufficiently small. We point out that, in the present work, the notion $x>y$ does not mean $x \geq y$ and $x \neq y$. Rather, it is to be understood as $x-y \geq 0$ and $x-y$ invertible. By [22, 1.2.38] and [24, Prop. 3.2] we have:

Lemma 1. Let $x \in \widetilde{\mathbb{R}}$. Then the following are equivalent:

(i) $\quad x>0$.

(ii) For each representative $\left(x_{\varepsilon}\right)$ of $x$ there exists some $\varepsilon_{0}$ and some $m$ such that $x_{\varepsilon}>\varepsilon^{m}$ for all $\varepsilon<\varepsilon_{0}$.

(iii) For each representative $\left(x_{\varepsilon}\right)$ of $x$ there exists some $\varepsilon_{0}$ such that $x_{\varepsilon}>0$ for all $\varepsilon<\varepsilon_{0}$.

We shall use the notation $\mathrm{d} \varepsilon^{m}:=\left[\varepsilon^{m}\right] \in \widetilde{\mathbb{R}}$ for any $m \in \mathbb{R}$. Hence $x>0$ is equivalent to $x \geq \mathrm{d} \varepsilon^{m}$ for some $m>0$. If $\mathcal{P}(\varepsilon)$ is a property of $\varepsilon \in I$, we will also sometimes use the notation $\forall^{0} \varepsilon: \mathcal{P}(\varepsilon)$ to denote $\exists \varepsilon_{0} \in I \forall \varepsilon \in\left(0, \varepsilon_{0}\right]: \mathcal{P}(\varepsilon)$.

The space of compactly supported generalized points $\widetilde{\Omega}_{c}$ is defined by $\Omega_{c} / \sim$, where $\Omega_{c}:=\left\{\left(x_{\varepsilon}\right) \in \Omega^{I} \mid \exists K \Subset \Omega \forall^{0} \varepsilon: x_{\varepsilon} \in K\right\}$ and $\sim$ is the same equivalence relation as in the case of $\widetilde{\Omega}$.

Concerning intervals, we use the following notations: $[a, b]:=\{x \in \widetilde{\mathbb{R}} \mid a \leq x \leq$ $b\},[a, b]_{\mathbb{R}}:=[a, b] \cap \mathbb{R}$. Also, for $x, y \in \widetilde{\mathbb{R}}^{n}$ we write $x \approx y$ if $x-y$ is infinitesimal, i.e. if $|x-y| \leq r$ for all $r \in \mathbb{R}_{>0}$.

As already indicated above, the natural topology for Colombeau-type spaces is the so-called sharp topology $([7,29,28,4,5,23,16])$. This topology is generated 
by balls $B_{\rho}(x)=\left\{y \in \widetilde{\mathbb{R}}^{n}|| y-x \mid<\rho\right\}$, where $|-|$ is the natural extension of the Euclidean norm to $\widetilde{\mathbb{R}}^{n},\left|\left[x_{\varepsilon}\right]\right|:=\left[\left|x_{\varepsilon}\right|\right] \in \widetilde{\mathbb{R}}$, and $\rho \in \widetilde{\mathbb{R}}_{>0}$ is strictly positive ([2, 3, 17]). For Euclidean balls, we will write $B_{\rho}^{\mathrm{E}}(x)=\left\{y \in \mathbb{R}^{n}|| y-x \mid<\rho\right\}$. On the other hand, the so-called Fermat-topology on $\widetilde{\mathbb{R}}^{n}$ (see $[17,18]$ ) is generated by the balls $B_{r}(x)$ for $x \in \widetilde{\mathbb{R}}^{n}$ and $r \in \mathbb{R}_{>0}$. Originally, the sharp topology was introduced using an ultrametric as follows: The map

$$
\begin{aligned}
& v: \mathbb{R}_{M} \longrightarrow(-\infty, \infty] \\
& v\left(\left(u_{\varepsilon}\right)\right):=\sup \left\{b \in \mathbb{R}|| u_{\varepsilon} \mid=O\left(\varepsilon^{b}\right)\right\} .
\end{aligned}
$$

gives a pseudovaluation on $\widetilde{\mathbb{R}}$. Then setting $|-|_{e}: \widetilde{\mathbb{R}} \rightarrow[0, \infty),|u|_{e}:=\exp (-v(u))$ provides a translation-invariant complete ultrametric

$$
\begin{aligned}
& d_{s}: \widetilde{\mathbb{R}} \times \widetilde{\mathbb{R}} \longrightarrow \mathbb{R}_{+} \\
& d_{s}(u, v):=|u-v|_{e}
\end{aligned}
$$

on $\widetilde{\mathbb{R}}$, which induces the sharp topology on $\widetilde{\mathbb{R}}$.

Garetto in [11, 12] extended the above construction to arbitrary locally convex spaces by functorially assigning a space of CGF $\mathcal{G}_{E}$ to any given locally convex space $E$. In this approach, the seminorms of $E$ are used to define pseudovaluations which induce a generalized locally convex topology on the $\widetilde{\mathbb{C}}$-module $\mathcal{G}_{E}$, again called sharp topology. In the present paper, we will exclusively work with $\widetilde{\mathbb{R}}$-modules. We note, however, that all our constructions trivially carry over to the $\widetilde{\mathbb{C}}$-case.

For any $S \subseteq I, e_{S}$ denotes the equivalence class in $\widetilde{\mathbb{R}}$ of the characteristic function of $S$ (cf. [4, 30]). Any $e_{S}$ is an idempotent, and $e_{S}+e_{S^{c}}=1$. Also, $e_{S} \neq 0$ if and only if $0 \in \bar{S}$. For any subset $A$ of $\widetilde{\mathbb{R}}^{n}$, its interleaving (cf. [27]) is defined as

$$
\operatorname{interl}(A):=\left\{\sum_{j=1}^{m} e_{S_{j}} a_{j} \mid m \in \mathbb{N},\left\{S_{1}, \ldots, S_{m}\right\} \text { a partition of } I, a_{j} \in A\right\} .
$$

If $\left(A_{\varepsilon}\right)$ is a net of subsets of $\mathbb{R}^{n}$ then the internal set $([27,31])$ generated by $\left(A_{\varepsilon}\right)$ is

$$
\left[A_{\varepsilon}\right]=\left\{\left[x_{\varepsilon}\right] \in \widetilde{\mathbb{R}}^{n} \mid x_{\varepsilon} \in A_{\varepsilon} \text { for } \varepsilon \text { small }\right\},
$$

and the strongly internal set $([18])$ generated by $\left(A_{\varepsilon}\right)$ is

$$
\left\langle A_{\varepsilon}\right\rangle:=\left\{\left[x_{\varepsilon}\right] \in \widetilde{\mathbb{R}}^{n} \mid x_{\varepsilon} \in_{\varepsilon} A_{\varepsilon}\right\} .
$$

Here, $x_{\varepsilon} \in_{\varepsilon} A_{\varepsilon}$ means that $x_{\varepsilon} \in A_{\varepsilon}$ for $\varepsilon$ small and that the same property holds for any representative of $\left[x_{\varepsilon}\right]$. The net $\left(A_{\varepsilon}\right)$ is called sharply bounded if there exists some $N \in \mathbb{R}_{>0}$ such that for $\varepsilon$ sufficiently small we have $\sup _{x \in A_{\varepsilon}}|x| \leq \varepsilon^{-N}$. Equivalently, we have that $\left(A_{\varepsilon}\right)$ is sharply bounded if there exists $\rho \in \widetilde{\mathbb{R}}_{>0}$ such that $\left[A_{\varepsilon}\right] \subseteq B_{\rho}(0)$. 
Finally, given $X \subseteq \widetilde{\mathbb{R}}^{n}$ and $Y \subseteq \widetilde{\mathbb{R}}^{d}$, then (see [18])

$$
f: X \longrightarrow Y \text { is a generalized smooth function (GSF) }
$$

if there exists a net $u_{\varepsilon} \in \mathcal{C}^{\infty}\left(\Omega_{\varepsilon}, \mathbb{R}^{d}\right)$ defining $f$ in the sense that $X \subseteq\left\langle\Omega_{\varepsilon}\right\rangle$, $f\left(\left[x_{\varepsilon}\right]\right)=\left[u_{\varepsilon}\left(x_{\varepsilon}\right)\right] \in Y$ and $\left(\partial^{\alpha} u_{\varepsilon}\left(x_{\varepsilon}\right)\right) \in \mathbb{R}_{M}^{d}$ for all $x=\left[x_{\varepsilon}\right] \in X$ and all $\alpha \in \mathbb{N}^{n}$. The space of GSF from $X$ to $Y$ is denoted by $\mathcal{G C}^{\infty}(X, Y)$ (in contrast to [18], where the notation $\widetilde{\mathcal{G}}(X, Y)$ was used). GSF are a natural generalization of CGF to general domains. In particular, for any $\Omega \subseteq \mathbb{R}^{n}$ open, $\mathcal{G C}^{\infty}\left(\widetilde{\Omega}_{c}\right) \simeq \mathcal{G}^{s}(\Omega)$. GSF on subsets of $\widetilde{\mathbb{R}}^{n}$, endowed with the sharp topology, form a sub-category of the category of topological spaces. In particular, they can be composed unrestrictedly.

\section{A NEW NOTION OF COMPACT SUbSET FOR NONLINEAR GENERALIZED} FUNCTIONS

Even though the intervals $[a, b] \subseteq \widetilde{\mathbb{R}}, a, b \in \mathbb{R}$, are neither compact in the sharp nor in the Fermat topology (see [18, Thm. 25]), analogously to the case of smooth functions, a GSF satisfies an extreme value theorem on such sets. In fact, we have:

Proposition 2. Let $f \in \mathcal{G C}^{\infty}(X, \widetilde{\mathbb{R}})$ be a generalized smooth function defined on the subset $X$ of $\widetilde{\mathbb{R}}^{n}$. Let $\emptyset \neq K=\left[K_{\varepsilon}\right] \subseteq X$ be an internal set generated by a sharply bounded net $\left(K_{\varepsilon}\right)$ of compact sets $K_{\varepsilon} \Subset \mathbb{R}^{n}$, then

$$
\exists m, M \in K \forall x \in K: \quad f(m) \leq f(x) \leq f(M) .
$$

Proof. By [18, Lem. 28], $f$ can be represented by a net $u_{\varepsilon} \in \mathcal{C}^{\infty}\left(\mathbb{R}^{n}, \mathbb{R}^{d}\right)$. Since $K \neq$ $\emptyset$, for $\varepsilon$ sufficiently small, say for $\varepsilon \in\left(0, \varepsilon_{0}\right], K_{\varepsilon}$ is non-empty and, by assumption, it is also compact. For all $\varepsilon \in\left(0, \varepsilon_{0}\right]$ we have

$$
\exists m_{\varepsilon}, M_{\varepsilon} \in K_{\varepsilon} \forall x \in K_{\varepsilon}: u_{\varepsilon}\left(m_{\varepsilon}\right) \leq u_{\varepsilon}(x) \leq u_{\varepsilon}\left(M_{\varepsilon}\right) .
$$

Since the net $\left(K_{\varepsilon}\right)$ is sharply bounded, both the nets $\left(m_{\varepsilon}\right)$ and $\left(M_{\varepsilon}\right)$ are moderate. Therefore $m=\left[m_{\varepsilon}\right], M=\left[M_{\varepsilon}\right] \in K \subseteq X$. Take any $x \in\left[K_{\varepsilon}\right]$, then there exists a representative $\left(x_{\varepsilon}\right)$ such that $x_{\varepsilon} \in K_{\varepsilon}$ for $\varepsilon$ small. Therefore $f(m)=\left[u_{\varepsilon}\left(m_{\varepsilon}\right)\right] \leq$ $\left[u_{\varepsilon}\left(x_{\varepsilon}\right)\right]=f(x) \leq f(M)$.

We shall use the assumptions on $K$ and $\left(K_{\varepsilon}\right)$ given in this theorem to introduce a new notion of "compact subset" which behaves better than the usual classical notion of compactness in the sharp topology.

Definition 3. A subset $K$ of $\widetilde{\mathbb{R}}^{n}$ is called functionally compact, denoted by $K \Subset_{\mathrm{f}}$ $\widetilde{\mathbb{R}}^{n}$, if there exists a net $\left(K_{\varepsilon}\right)$ such that

(i) $\quad K=\left[K_{\varepsilon}\right] \subseteq \widetilde{\mathbb{R}}^{n}$

(ii) $\quad\left(K_{\varepsilon}\right)$ is sharply bounded

(iii) $\forall \varepsilon \in I: K_{\varepsilon} \Subset \mathbb{R}^{n}$ 
If, in addition, $K \subseteq U \subseteq \widetilde{\mathbb{R}}^{n}$ then we write $K \Subset_{\mathrm{f}} U$. Finally, we write $\left[K_{\varepsilon}\right] \Subset_{\mathrm{f}} U$ if (ii), (iii) and $\left[K_{\varepsilon}\right] \subseteq U$ hold.

We note that in (iii) it suffices to ask that $K_{\varepsilon}$ be closed since it is bounded by (ii), at least for $\varepsilon$ small. In fact, we have:

Lemma 4. A subset $K$ of $\widetilde{\mathbb{R}}^{n}$ is functionally compact if and only if it is internal and sharply bounded.

Proof. By [27, Lemma 2.4 and Cor. 2.2], every sharply bounded internal set $K$ has a sharply bounded representative $\left(K_{\varepsilon}\right)$ consisting of closed (hence compact) subsets of $\mathbb{R}^{n}$.

We motivate the name functionally compact subset by anticipating that on this type of subsets, GSF have properties very similar to those that ordinary smooth functions have on standard compact sets.

Remark 5.

(i) By [27, Prop. 2.3], any internal set $K=\left[K_{\varepsilon}\right]$ is closed in the sharp topology. In particular, the open interval $(0,1) \subseteq \widetilde{\mathbb{R}}$ is not functionally compact since it is not closed.

(ii) If $H \Subset \mathbb{R}^{n}$ is a non-empty ordinary compact set, then $\widetilde{H}=[H]$ is functionally compact. In particular, $[0,1]=\widetilde{[0,1]_{\mathbb{R}}}=\left[[0,1]_{\mathbb{R}}\right]$ is functionally compact.

(iii) The empty set $\emptyset=\widetilde{\emptyset} \Subset_{\mathrm{f}} \widetilde{\mathbb{R}}$.

(iv) By Lemma $4, \widetilde{\mathbb{R}}^{n}$ is not functionally compact since it is not sharply bounded.

(v) The set of compactly supported points $\widetilde{\mathbb{R}}_{c}$ is not functionally compact because the GSF $f(x)=x$ does not satisfy the conclusion (2.1) of Prop. 2.

We start the study of functionally compact sets by proving suitable generalizations of theorems from classical analysis.

Theorem 6. Let $K \subseteq X \subseteq \widetilde{\mathbb{R}}^{n}, f \in \mathcal{G C} \mathcal{C}^{\infty}\left(X, \widetilde{\mathbb{R}}^{d}\right)$. Then $K \Subset_{f} \widetilde{\mathbb{R}}^{n}$ implies $f(K) \Subset_{f}$ $\widetilde{\mathbb{R}}^{d}$.

Proof. Let $\left(K_{\varepsilon}\right)$ be as in Def. 3 and let the GSF $f$ be defined by the net $u_{\varepsilon} \in$ $\mathcal{C}^{\infty}\left(\mathbb{R}^{n}, \mathbb{R}^{d}\right)$. Let us first prove that $f(K)=\left[u_{\varepsilon}\left(K_{\varepsilon}\right)\right]$. In fact, $y \in f(K)=f\left(\left[K_{\varepsilon}\right]\right)$ is equivalent to

$$
\exists\left(x_{\varepsilon}\right) \in \mathbb{R}_{M}^{n} \forall^{0} \varepsilon: x_{\varepsilon} \in K_{\varepsilon} \text { and } y=\left[u_{\varepsilon}\left(x_{\varepsilon}\right)\right] .
$$

This necessary entails $y \in\left[u_{\varepsilon}\left(K_{\varepsilon}\right)\right]$. Vice versa, if $y \in\left[u_{\varepsilon}\left(K_{\varepsilon}\right)\right]$, then there exists $\left(y_{\varepsilon}\right) \in \mathbb{R}_{M}^{d}$ such that $y_{\varepsilon} \in u_{\varepsilon}\left(K_{\varepsilon}\right)$ for $\varepsilon$ small. Hence, for each of these $\varepsilon$ there also exists $x_{\varepsilon} \in K_{\varepsilon}$ such that $y_{\varepsilon}=u_{\varepsilon}\left(x_{\varepsilon}\right)$, which implies $y=\left[u_{\varepsilon}\left(x_{\varepsilon}\right)\right]$, i.e. (2.2) since $\left(K_{\varepsilon}\right)$ is sharply bounded. Clearly, $u_{\varepsilon}\left(K_{\varepsilon}\right) \Subset \mathbb{R}^{d}$, so it remains to prove that the 
net $\left(u_{\varepsilon}\left(K_{\varepsilon}\right)\right)$ is sharply bounded. If $\forall \varepsilon_{0} \exists \varepsilon \leq \varepsilon_{0}: K_{\varepsilon}=\emptyset$, then $\left[K_{\varepsilon}\right]=K=\emptyset$, so $f(K)=\emptyset$ and the conclusion is trivial. Otherwise, assume that $K_{\varepsilon} \neq \emptyset$ for $\varepsilon \leq \varepsilon_{0}$ and proceed by contradiction assuming that

$$
\forall k \in \mathbb{N} \exists\left(\varepsilon_{k n}\right)_{n} \downarrow 0 \forall n \exists y_{k n} \in u_{\varepsilon_{k n}}\left(K_{\varepsilon_{k n}}\right):\left|y_{k n}\right|>\varepsilon_{k n}^{-k} .
$$

We can write $y_{k n}=u_{\varepsilon_{k n}}\left(x_{k n}\right)$ for some $x_{k n} \in K_{\varepsilon_{k n}}$. Next, set $\varepsilon_{0}:=\varepsilon_{00}$ and for $k>0$ pick $n_{k}$ such that $\varepsilon_{k n_{k}}<\min \left(\frac{1}{k}, \varepsilon_{k-1}\right)$ and set $\varepsilon_{k}:=\varepsilon_{k n_{k}}$. Take any $\bar{x}_{\varepsilon} \in K_{\varepsilon}$ for each $\varepsilon \leq \varepsilon_{0}$, and set $x_{\varepsilon}:=x_{k n_{k}}$ if $\varepsilon=\varepsilon_{k}$ and $x_{\varepsilon}:=\bar{x}_{\varepsilon}$ otherwise. Then $x_{\varepsilon} \in K_{\varepsilon}$ for $\varepsilon \leq \varepsilon_{0}$, so $x=\left[x_{\varepsilon}\right] \in K \subseteq X$ and $\left(u_{\varepsilon}\left(x_{\varepsilon}\right)\right) \in \mathbb{R}_{M}^{d}$ by the definition of GSF, which contradicts (2.3).

As a corollary of this theorem and Rem. (5).(ii) we get

Corollary 7. If $a, b \in \widetilde{\mathbb{R}}$ and $a \leq b$, then $[a, b] \Subset_{f} \widetilde{\mathbb{R}}$.

Let us note that $a, b \in \widetilde{\mathbb{R}}$ can also be infinite, e.g. $a=\left[-\varepsilon^{-N}\right], b=\left[\varepsilon^{-M}\right]$ or $a=\left[\varepsilon^{-N}\right], b=\left[\varepsilon^{-M}\right]$ with $M>N$.

Lemma 8. Let $K, H \Subset_{f} \widetilde{\mathbb{R}}^{n}$, then we have:

(i) $\quad K \cup H \subseteq \operatorname{interl}(K \cup H) \Subset_{f} \widetilde{\mathbb{R}}^{n}$

(ii) If $K \cup H$ is internal, then it is functionally compact

(iii) If $K \cap H$ is internal, then it is functionally compact.

Proof. (i) follows from [27, Prop. 2.8] which implies $K \cup H \subseteq \operatorname{interl}(K \cup H)=$ $\left[K_{\varepsilon} \cup H_{\varepsilon}\right]$, where the nets $\left(K_{\varepsilon}\right)$ and $\left(H_{\varepsilon}\right)$ satisfy Def. 3. Property (ii) follows from [27, Lemma 2.7] which implies that if the union of internal sets is internal, then it is equal to its interleaving. Property (iii) is a consequence of Lemma 4.

If $H \subseteq K \Subset_{\mathrm{f}} \widetilde{\mathbb{R}}^{n}$, then also $H$ is sharply bounded. So, another consequence of Lemma 4 is the following:

Corollary 9. Let $H \subseteq K \Subset_{f} \widetilde{\mathbb{R}}^{n}$, then $H$ internal implies $H \Subset_{f} \widetilde{\mathbb{R}}^{n}$.

Finally, in the following result we consider the product of functionally compact sets:

Proposition 10. Let $K \Subset_{f} \widetilde{\mathbb{R}}^{n}$ and $H \Subset_{f} \widetilde{\mathbb{R}}^{d}$, then $K \times H \Subset_{f} \widetilde{\mathbb{R}}^{n+d}$. In particular, if $a_{i} \leq b_{i}$ for $i=1, \ldots, n$, then $\prod_{i=1}^{n}\left[a_{i}, b_{i}\right] \Subset_{f} \widetilde{\mathbb{R}}^{n}$.

Proof. From [27, Prop. 2.13] if follows that $K \times H$ is internal, in fact for $K=\left[K_{\varepsilon}\right]$, $H=\left[H_{\varepsilon}\right], K \times H=\left[K_{\varepsilon} \times H_{\varepsilon}\right]$. From this representation it immediately follows that $H \times K$ is sharply bounded as well, so Lemma 4 gives the claim. 


\section{Compactly Supported Generalized Smooth FunCtions}

Our main goal in this section is to define and study an analogue within GSF of the space $\mathcal{D}_{K}(\Omega)$ of smooth functions supported in a fixed compact set $K \Subset \Omega$. In order to define this space, we first try to define the concept of support of a GSF. Clearly, if $\varphi \in \mathcal{D}_{[-a, a]_{\mathbb{R}}}(\mathbb{R})$, one would expect that $\varphi$ should have compact support also if we think of $\varphi$ as a GSF. In fact, $\operatorname{supp}(\varphi)$ should be contained in $[-a, a] \Subset_{\mathrm{f}} \widetilde{\mathbb{R}}$. Already this basic requirement implies that if $f \in \mathcal{G C}^{\infty}(X, Y)$, the natural definition

$$
S(f):=X \backslash \bigcup\left\{B_{\rho}(x) \cap X\left|x \in X, \rho \in \widetilde{\mathbb{R}}_{>0}, f\right|_{B_{\rho}(x) \cap X}=0\right\}
$$

doesn't fit with our intuition. Indeed, if we take the aforementioned $\varphi$ so that $\varphi(0)=1$, and $S \subseteq(0,1]$ such that $0 \in \bar{S}$ and $0 \in \overline{S^{c}}$, then $\varphi\left(e_{S} \mathrm{~d} \varepsilon^{-1}\right)=e_{S^{c}} \neq 0$ and $e_{S} \mathrm{~d} \varepsilon^{-1} \in S(\varphi) \backslash[-a, a]$. This motivates the following

Definition 11. Let $X \subseteq \widetilde{\mathbb{R}}^{n}, Y \subseteq \widetilde{\mathbb{R}}^{d}$ and $f \in \mathcal{G C}^{\infty}(X, Y)$, then

$$
\operatorname{supp}(f):=\overline{\{x \in X|| f(x) \mid>0\}}
$$

where here $\overline{(-)}$ denotes the relative closure in $X$ with respect to the sharp topology.

Using this concept, we have $\operatorname{supp}(\varphi) \subseteq[-a, a]$. In fact, $|\varphi(x)|=\left[\left|\varphi\left(x_{\varepsilon}\right)\right|\right]>0$ implies $\left|\varphi\left(x_{\varepsilon}\right)\right|>\varepsilon^{q}$ for some $q \in \mathbb{R}_{>0}$ and for $\varepsilon$ small, and hence $x_{\varepsilon} \in[-a, a]_{\mathbb{R}}$.

Remark 12.

(i) In the setting of Colombeau algebras, one usually defines the support of some $f \in \mathcal{G}^{s}(\Omega)$ as a subset of $\Omega \subseteq \mathbb{R}^{n}$, i.e., as a set of classical points, namely as $\operatorname{supp}_{\mathcal{G}^{s}}(f):=\Omega \backslash \bigcup\left\{B_{r}(x) \cap \Omega\left|x \in \Omega, r \in \mathbb{R}_{>0}, f\right|_{B_{r}(x) \cap \Omega}=0\right\}$, where the last equality has to be understood in $\mathcal{G}^{s}\left(B_{r}(x) \cap \Omega\right)$. Using $X=\widetilde{\Omega}_{c}$ as the natural domain of any $f \in \mathcal{G}^{s}(\Omega)$ (cf. [18, Thm. 37]), it is then immediate that $\operatorname{supp}(f) \cap \Omega \subseteq \operatorname{supp}_{\mathcal{G}^{s}}(f)$.

(ii) Let $u \in \mathcal{D}^{\prime}(\Omega)$ be a Schwartz distribution and denote by $\iota: \mathcal{D}^{\prime}(\Omega) \rightarrow$ $\mathcal{G C}^{\infty}\left(\widetilde{\Omega}_{c}, \mathbb{R}\right)$ a standard embedding via convolution. Then $\operatorname{supp}(\iota(u)) \cap \Omega \subseteq$ $\operatorname{supp}(u)$, as follows from (i) and the fact that $\iota$ is a sheaf-morphism.

(iii) Assume that the embedding $\iota: \mathcal{D}^{\prime}(\Omega) \rightarrow \mathcal{G C}^{\infty}\left(\widetilde{\Omega}_{c}, \mathbb{R}\right)$ has been defined by using a mollifier $\rho \in \mathcal{S}\left(\mathbb{R}^{n}\right)$ which is identically equal to 1 in the ball $B_{p}^{\mathrm{E}}(0)$, $p \in \mathbb{R}_{>0}$. Then $\delta(x)=\mathrm{d} \varepsilon^{-n}$ for each $x \in B_{p \cdot \mathrm{d} \varepsilon}(0)$ and hence $B_{p \cdot \mathrm{d} \varepsilon}(0) \subseteq$ $\operatorname{supp}(\iota(\delta))$, whereas $\operatorname{supp}(\iota(\delta)) \cap \mathbb{R}^{n}=\{0\}$.

(iv) In general, $\operatorname{supp}(f)$ is not an internal set because it is not generally closed by finite interleaving (see [27, Lem. 2.7]). Consider e.g. $X$ with only near standard points and $f \in \mathcal{G C}^{\infty}(X, \widetilde{\mathbb{R}})$ which is strictly positive on two disjoint intervals. However, if $X$ itself is closed under finite interleaving then so is $\operatorname{supp}(f)$. 
If $\left(u_{\varepsilon}\right)$ defines $f \in \mathcal{G C}^{\infty}(X, Y)$, the internal set $\left[\operatorname{supp}\left(u_{\varepsilon}\right)\right]$ is not intrinsically defined since it depends on the defining net $\left(u_{\varepsilon}\right)$. Consider, e.g., $u_{\varepsilon}(x):=\varphi(x)+$ $\varepsilon^{1 / \varepsilon}>0$ where $\varphi \in \mathcal{C}^{\infty}\left(\mathbb{R}, \mathbb{R}_{\geq 0}\right)$.

In our further analysis we will repeatedly make use of the following notion:

Definition 13. For $A \subseteq \widetilde{\mathbb{R}}^{n}$ we call the set

$$
\operatorname{ext}(A):=\left\{x \in \widetilde{\mathbb{R}}^{n}|\forall a \in A:| x-a \mid>0\right\}
$$

the strong exterior of $A$.

This set can also be described in the following way:

Lemma 14. If $A \subseteq \widetilde{\mathbb{R}}^{n}$, then $\operatorname{ext}(A)=\left\{x \in \widetilde{\mathbb{R}}^{n} \mid \forall S \subseteq I: e_{S} \neq 0 \Rightarrow x e_{S} \notin A e_{S}\right\}$.

Proof. $\subseteq$ : Let $x=\left[x_{\varepsilon}\right] \in \operatorname{ext}(A)$ and suppose that there exists some $S \subseteq I$ with $e_{S} \neq 0$ and some $a=\left[a_{\varepsilon}\right] \in A$ such that $x e_{S}=a e_{S}$. Then there exists some $q>0$ such that $\left|x_{\varepsilon}-a_{\varepsilon}\right|>\varepsilon^{q}$ for $\varepsilon$ small. However, $x e_{S}=a e_{S}$ implies that $\left|x_{\varepsilon}-a_{\varepsilon}\right|=O\left(\varepsilon^{q+1}\right)$ for $\varepsilon \rightarrow 0, \varepsilon \in S$, a contradiction.

: If there exists some $a=\left[a_{\varepsilon}\right] \in A$ such that $|x-a| \ngtr 0$ then there is a sequence $\varepsilon_{k} \downarrow 0$ with $\left|x_{\varepsilon_{k}}-a_{\varepsilon_{k}}\right|<\varepsilon_{k}^{k}$ for all $k$. Letting $S:=\left\{\varepsilon_{k} \mid k \in \mathbb{N}\right\}$ implies $x e_{S}=a e_{S}$.

For non-trivial internal sets we have the following characterization of the strong exterior:

Lemma 15. Let $\emptyset \neq\left[K_{\varepsilon}\right]=K \subseteq \widetilde{\mathbb{R}}^{n}$. Then

$$
\operatorname{ext}(K)=\left\langle K_{\varepsilon}^{c}\right\rangle
$$

Proof. Suppose first that $x \in\left\langle K_{\varepsilon}^{c}\right\rangle$ and let $S \subseteq I$ with $e_{S} \neq 0$. Suppose that there existed some $a \in K$ with $x e_{S}=a e_{S}$. Since $a \in K$ there exists a representative $\left(a_{\varepsilon}\right)$ of $a$ with $a_{\varepsilon} \in K_{\varepsilon}$ for all $\varepsilon$. Then $x e_{S}=a e_{S}$ implies that there exists a representative $\left(x_{\varepsilon}\right)$ of $x$ and a sequence $\varepsilon_{k} \downarrow 0$ in $S$ with $x_{\varepsilon_{k}}=a_{\varepsilon_{k}} \in K_{\varepsilon_{k}}$ for all $k$. But this contradicts the fact that $x \in\left\langle K_{\varepsilon}^{c}\right\rangle$.

Conversely, if $x \notin\left\langle K_{\varepsilon}^{c}\right\rangle$ then there exists a representative $\left(x_{\varepsilon}\right)$ of $x$ and a sequence $\varepsilon_{m} \downarrow 0$ with $x_{\varepsilon_{m}} \in K_{\varepsilon_{m}}$ for all $m$. Since $K \neq \emptyset$, there exists some $w=\left[w_{\varepsilon}\right] \in K$ with $w_{\varepsilon} \in K_{\varepsilon}$ for all $\varepsilon$. Now let

$$
a_{\varepsilon}:= \begin{cases}x_{\varepsilon_{m}} & \text { if } \varepsilon=\varepsilon_{m} \\ w_{\varepsilon} & \text { otherwise }\end{cases}
$$

and set $S:=\left\{\varepsilon_{m} \mid m \in \mathbb{N}\right\}$. Then $a=\left[a_{\varepsilon}\right] \in\left[K_{\varepsilon}\right]$ and $x e_{S}=a e_{S}$ by construction. Thus $x e_{S} \in K e_{S}$, and so $x \notin \operatorname{ext}(K)$.

As an immediate conclusion we obtain: 
Corollary 16. Let $K=\left[K_{\varepsilon}\right]=\left[L_{\varepsilon}\right] \neq \emptyset$. Then $\left\langle K_{\varepsilon}^{c}\right\rangle=\left\langle L_{\varepsilon}^{c}\right\rangle$.

The next result relates the support of a GSF to the exterior of certain internal sets. To formulate it concisely, we introduce the following notations: Denote by $\mathcal{K}_{f}$ the set of all internal $\emptyset \neq K \subseteq \widetilde{\mathbb{R}}^{n}$ with $\operatorname{ext}(K) \neq \emptyset$ and such that there exists a net $u_{\varepsilon} \in \mathcal{C}^{\infty}\left(\mathbb{R}^{n}, \mathbb{R}^{d}\right)$ that defines $f$ and such that $\left[u_{\varepsilon}\left(x_{\varepsilon}\right)\right]=0$ for all $\left[x_{\varepsilon}\right] \in \operatorname{ext}(\mathrm{K})$. Also, denote by $\mathcal{H}_{f}$ the set of all the internal sets of the form $K=\left[\operatorname{supp}\left(u_{\varepsilon}\right)\right] \subseteq \widetilde{\mathbb{R}}^{n}$ for some net $u_{\varepsilon} \in \mathcal{C}^{\infty}\left(\mathbb{R}^{n}, \mathbb{R}^{d}\right)$ that defines $f$ and such that both $K$ and $\operatorname{ext}(K)$ are non empty.

Then we have:

Lemma 17. Let $X \subseteq \widetilde{\mathbb{R}}^{n}, Y \subseteq \widetilde{\mathbb{R}}^{d}$ and $f \in \mathcal{G C}^{\infty}(X, Y)$. Then

$$
\operatorname{supp}(f) \subseteq X \cap \bigcap_{K \in \mathcal{K}_{f}} K \subseteq X \cap \bigcap_{K \in \mathcal{H}_{f}} K .
$$

Proof. Since $X \cap \bigcap_{K \in \mathcal{K}_{f}} K$ is a sharply closed subset of $X$, in order to show the first inclusion in (3.1), it suffices to prove that

$$
\{x \in X|| f(x) \mid>0\} \subseteq X \cap \bigcap_{K \in \mathcal{K}_{f}} K .
$$

Let $x \in X$ be such that $|f(x)|>0$, so that

$$
\exists r \in \mathbb{R}_{>0}:|f(x)|>\mathrm{d} \varepsilon^{r} .
$$

Let $K=\left[K_{\varepsilon}\right] \in \mathcal{K}_{f}$, and assume, by contradiction, that $x=\left[x_{\varepsilon}\right] \notin K$. We first prove that

$$
\exists q \in \mathbb{N}_{>r} \exists\left(\varepsilon_{k}\right)_{k \in \mathbb{N}} \downarrow 0 \forall k \in \mathbb{N}: B_{\varepsilon_{k}^{q}}^{\mathrm{E}}\left(x_{\varepsilon_{k}}\right) \subseteq K_{\varepsilon_{k}}^{c},
$$

where $r$ comes from (3.2). In fact, suppose to the contrary that

$$
\forall q \in \mathbb{N}_{>r} \exists \varepsilon_{q} \forall \varepsilon \leq \varepsilon_{q} \exists y_{\varepsilon}^{(q)} \in B_{\varepsilon^{q}}^{\mathrm{E}}\left(x_{\varepsilon}\right): y_{\varepsilon}^{(q)} \in K_{\varepsilon} .
$$

We may assume that $\left(\varepsilon_{q}\right)_{q \in \mathbb{N}} \downarrow 0$. Setting $\tilde{y}_{\varepsilon}:=y_{\varepsilon}^{(q)}$ for $\varepsilon \in\left(\varepsilon_{q+1}, \varepsilon_{q}\right]$, we have $x=\left[\tilde{y}_{\varepsilon}\right] \in K$, which contradicts $x \notin K$.

By assumption $\exists z=\left[z_{\varepsilon}\right] \in \operatorname{ext}(K)$, and hence

$$
\exists s \in \mathbb{N}_{>q} \forall^{0} \varepsilon: d\left(z_{\varepsilon}, K_{\varepsilon}\right)>\varepsilon^{s},
$$

where $q$ comes from (3.3). Using $\left(\varepsilon_{k}\right)_{k \in \mathbb{N}}$ from (3.3), we set $\tilde{x}_{\varepsilon}:=x_{\varepsilon_{k}}$ if $\varepsilon=\varepsilon_{k}$ and $\tilde{x}_{\varepsilon}:=z_{\varepsilon}$ otherwise. Then $\tilde{x}:=\left[\tilde{x}_{\varepsilon}\right] \in \operatorname{ext}(K)$. But $K \in \mathcal{K}_{f}$, so there exists a net $u_{\varepsilon} \in \mathcal{C}^{\infty}\left(\mathbb{R}^{n}, \mathbb{R}^{d}\right)$ that defines $f$ and such that $\left[u_{\varepsilon}\left(\tilde{x}_{\varepsilon}\right)\right]=0$. In particular, $\left|u_{\varepsilon_{k}}\left(\tilde{x}_{\varepsilon_{k}}\right)\right|=\left|u_{\varepsilon_{k}}\left(x_{\varepsilon_{k}}\right)\right|=O\left(\varepsilon_{k}^{2 s}\right)$ as $k \rightarrow+\infty$, which contradicts $|f(x)|=$ $\left[\left|u_{\varepsilon}\left(x_{\varepsilon}\right)\right|\right]>\mathrm{d} \varepsilon^{r}>\mathrm{d} \varepsilon^{s}$.

Turning now to the second inclusion, assume that $x \in X \backslash \bigcap_{K \in \mathcal{H}_{f}} K$, so that there exists a net $\left(u_{\varepsilon}\right)$ that defines $f$, with $\emptyset \neq K:=\left[\operatorname{supp}\left(u_{\varepsilon}\right)\right] \subseteq \widetilde{\mathbb{R}}^{n}$ and $\operatorname{ext}(K) \neq \emptyset$, 
but such that $x \notin K$. We want to show that $x \notin \bigcap_{K^{\prime} \in \mathcal{K}_{f}} K^{\prime}$. But for all $\left[y_{\varepsilon}\right] \in$ $\operatorname{ext}(K)=\left\langle\operatorname{supp}\left(u_{\varepsilon}\right)^{c}\right\rangle$, we have $y_{\varepsilon} \notin \operatorname{supp}\left(u_{\varepsilon}\right)$ for $\varepsilon$ small. Hence, $u_{\varepsilon}\left(y_{\varepsilon}\right)=0$ for these $\varepsilon$, and this yields $\left[u_{\varepsilon}\left(y_{\varepsilon}\right)\right]=0$. This proves that $K \in \mathcal{K}_{f}$, but $x \notin K$.

\section{Remark 18.}

(i) Using methods from Nonstandard Analysis, one can prove the converse of the first inclusion in (3.1) in the following case: If $X=\widetilde{\mathbb{R}}^{n}$ and the sharp interior of $\left\{x \in \widetilde{\mathbb{R}}^{n} \mid f(x)=0\right\}$ is non-empty, then

$$
\operatorname{supp}(f)=\bigcap_{K \in \mathcal{K}_{f}} K
$$

To see this, let $x \in \bigcap_{K \in \mathcal{K}_{f}} K$ and choose some $z$ in the interior of $\left\{y \in \widetilde{\mathbb{R}}^{n}\right.$ $\mid f(y)=0\}$ and some $q>0$ such that $B_{d \varepsilon^{q}}(z) \subseteq\left\{y \in \widetilde{\mathbb{R}}^{n} \mid f(y)=0\right\}$. Given a representative $\left(v_{\varepsilon}\right)$ of $f$, let $\left(\chi_{\varepsilon}\right)$ be a moderate net of smooth functions such that $\chi_{\varepsilon}$ vanishes on $B_{\varepsilon^{q+2}}^{\mathrm{E}}\left(z_{\varepsilon}\right)$ and is identically equal to 1 on $\mathbb{R}^{n} \backslash B_{\varepsilon^{q+1}}^{\mathrm{E}}\left(z_{\varepsilon}\right)$. Then setting $u_{\varepsilon}:=\chi_{\varepsilon} v_{\varepsilon}$ gives a new representative of $f$ such that $\left[\left\{y \in \mathbb{R}^{n} \mid\right.\right.$ $\left.u_{\varepsilon}(y)=0\right\}$ ] has non-empty sharp interior. Let $m_{\varepsilon} \in \mathbb{N}, m_{\varepsilon} \rightarrow \infty$ as $\varepsilon \rightarrow 0$. Then set $K_{\varepsilon}:=\left\{y \in \mathbb{R}^{n}|| u_{\varepsilon}(y) \mid \geq \varepsilon^{m_{\varepsilon}}\right\}$ and $K:=\left[K_{\varepsilon}\right]$. It follows that $\emptyset \neq \operatorname{ext}(K) \subseteq\left\{y \in \mathbb{R}^{n} \mid f(y)=0\right\}$, so $x \in K$. Fix any $k \in \mathbb{N}$. Now using nonstandard notation, letting $\rho:=[\varepsilon], u:=\left[u_{\varepsilon}\right]$, and fixing a representative of $x$, which we temporarily simply denote by $x$, the above in particular implies

$$
{ }^{*} \mathbb{N}_{\infty} \subseteq\left\{m \in{ }^{*} \mathbb{N}\left|\exists y_{k} \in{ }^{*} \mathbb{R}^{n}:\right| y_{k}-x\left|\leq \rho^{k} \wedge\right| u\left(y_{k}\right) \mid \geq \rho^{m}\right\} .
$$

By the underspill principle, therefore, there also exists some $m \in \mathbb{N}$ and some $y_{k} \in{ }^{*} \mathbb{R}^{n}$ such that $\left|y_{k}-x\right| \leq \rho^{k}$ and $\left|u\left(y_{k}\right)\right| \geq \rho^{m}$. Taking equivalence classes of these nets, it follows that there exist $y_{k} \in \widetilde{\mathbb{R}}^{n}$ such that $\left|y_{k}-x\right| \leq \mathrm{d} \varepsilon^{k}$ and $\left|f\left(y_{k}\right)\right|>0$. Since $y_{k} \rightarrow x$ in the sharp topology, this implies that $x \in \operatorname{supp}(f)$.

(ii) The assumption $\operatorname{ext}(K) \neq \emptyset$ in the definition of $\mathcal{K}_{f}$ is essential. To illustrate this, take $\varphi$ as defined before Def. 11, pick any sequence $\varepsilon_{k} \downarrow 0$ and set $K_{\varepsilon}:=\{0\}$ for $\varepsilon=\varepsilon_{k}$, and $K_{\varepsilon}=\mathbb{R}^{n}$ otherwise. Then $\operatorname{ext}(K)=\emptyset$, and obviously $\operatorname{supp}(\varphi) \nsubseteq K$.

(iii) The question of whether the reverse of the last inclusion in (3.1) also holds remains open.

In the following section we shall see that even though the notion of support introduced above may not be entirely satisfactory, there nevertheless is a very convenient notion of being compactly supported for GSF. 
3.1. The spaces $\mathcal{G D}_{K}(U, Y)$ and $\mathcal{G D}(U, Y)$. A frequently used idea to solve problems like the previous ones comes by considering a family of GSF having "good representatives", i.e. possessing a defining net $\left(u_{\varepsilon}\right)$ that conforms to our intuition and includes the examples we have in mind. In the following, we denote by $\left(u^{1}, \ldots, u^{d}\right)$ the components of a function $u$ which takes values, e.g., in $\mathbb{R}^{d}$.

Definition 19. Let $\emptyset \neq K \Subset_{\mathrm{f}} U \subseteq \widetilde{\mathbb{R}}^{n}$ and $Y \subseteq \widetilde{\mathbb{R}}^{d}$, then we say that $f$ is a $G S F$ compactly supported in $K$, and we write

$$
f \in \mathcal{G D}_{K}(U, Y)
$$

if $f \in \mathcal{G C}^{\infty}(U, Y)$ and there exists a net $\left(u_{\varepsilon}\right)$ such that:

(i) $\quad\left(u_{\varepsilon}\right)$ defines $f$, where $u_{\varepsilon} \in \mathcal{C}^{\infty}\left(\mathbb{R}^{n}, \mathbb{R}^{d}\right)$ for all $\varepsilon$.

(ii) $\forall \alpha \in \mathbb{N}^{n} \forall\left[x_{\varepsilon}\right] \in \operatorname{ext}(K):\left[\partial^{\alpha} u_{\varepsilon}\left(x_{\varepsilon}\right)\right]=0$.

Moreover, we set

$$
\mathcal{G D}(U, Y):=\bigcup_{\emptyset \neq K \Subset_{\mathrm{f}} U} \mathcal{G} \mathcal{D}_{K}(U, Y)
$$

We will simply use the symbols $\mathcal{G} \mathcal{D}_{K}(U)$ and $\mathcal{G D}(U)$ if $Y=\widetilde{\mathbb{R}}$.

Remark 20.

(i) Lemma 17 implies that if $f \in \mathcal{G D}_{K}(U, Y)$, then $\operatorname{supp}(f) \subseteq K$ because $K \in$ $\mathcal{K}_{f}$. The converse implication for an arbitrary subset $U$ remains an open problem. For the case $U=\widetilde{\mathbb{R}}^{n}$, and if $\operatorname{supp}(f)$ is not empty, see Thm. 27 below.

(ii) It is clear that, in general, another net defining $f: U \longrightarrow \widetilde{\mathbb{R}}^{d}$ will not necessarily satisfy (ii) of Def. 19 because such a net is not bound to have any particular behavior outside of $U$.

(iii) Set $K_{\varepsilon}:=\overline{B_{1}^{\mathrm{E}}(0)}$ and $L_{\varepsilon}:=K_{\varepsilon} \backslash B_{e^{-1 / \varepsilon}}^{\mathrm{E}}(0)$, then for the Hausdorff distance of $K_{\varepsilon}$ and $L_{\varepsilon}$ we obtain $d_{\mathcal{H}}\left(K_{\varepsilon}, L_{\varepsilon}\right)=e^{-\frac{1}{\varepsilon}}$. By [27, Cor. 2.10], it follows that $\left[K_{\varepsilon}\right]=\left[L_{\varepsilon}\right]$. If we consider a net of smooth functions such that $\left.u_{\varepsilon}\right|_{B_{1 / 2}^{\mathrm{E}}(0)}=1, u_{\varepsilon}{\mid \mathbb{R}^{2} \backslash K_{\varepsilon}}=0, u_{\varepsilon} \geq 0$, then $\sup _{y \in \mathbb{R}^{2} \backslash K_{\varepsilon}}\left|\partial^{\alpha} u_{\varepsilon}(y)\right|=0$ but $\sup _{y \in \mathbb{R}^{2} \backslash L_{\varepsilon}}\left|\partial^{\alpha} u_{\varepsilon}(y)\right|=1$. This motivates the use of the strongly internal set $\left\langle K_{\varepsilon}^{c}\right\rangle$ in (ii) instead of the simpler $\left[K_{\varepsilon}^{c}\right]$. Analogously, we can consider as $L_{\varepsilon}$ an $e^{-\frac{1}{\varepsilon}}$-mesh of points for $K_{\varepsilon}=\overline{B_{1}^{\mathrm{E}}(0)}$. $L_{\varepsilon}$ may also contain points in $K_{\varepsilon}^{c}$, but so that $d\left(x, K_{\varepsilon}\right)=e^{-\frac{1}{\varepsilon}}$. This example shows clearly that we need to be sufficiently "far" from $\partial K_{\varepsilon}$ to be sure that $\left[\partial^{\alpha} u_{\varepsilon}\left(x_{\varepsilon}\right)\right]=0$, i.e. at points $x=\left[x_{\varepsilon}\right]$ such that $\left[d\left(x_{\varepsilon}, K_{\varepsilon}\right)\right]>0$, as stated in (ii). Since ext $(K)$ is independent of the choice of representative of $K$ by Cor. 16, so is Def. 19. This is essential to prove the completeness of the spaces $\mathcal{G} \mathcal{D}_{K}(U)$ and $\mathcal{G D}(U)$.

The following result will turn out to be useful when proving results by contradiction in several instances below. It permits to restrict the analysis to only two cases: 
points in $K$ or in $\operatorname{ext}(K)$. To state it more clearly, we say that a generalized point $\left[y_{\varepsilon}\right]$ joins points of the sequence $\left(y_{k}\right)_{k \in \mathbb{N}}$ at $\left(\varepsilon_{k}\right)_{k \in \mathbb{N}}$ if $\forall N \in \mathbb{N} \exists k \geq N: y_{\varepsilon_{k}}=y_{k}$.

Lemma 21. Let $K=\left[K_{\varepsilon}\right] \Subset_{f} \widetilde{\mathbb{R}}^{n},\left(\varepsilon_{k}\right)_{k \in \mathbb{N}}$ a sequence in $(0,1]$ which strictly decreases to 0 , and $\left(y_{k}\right)_{k \in \mathbb{N}}$ a sequence in $\mathbb{R}^{n}$. For each $k \in \mathbb{N}$, let $x_{k} \in K_{\varepsilon_{k}}$ be such that $d\left(y_{k}, x_{k}\right)=d\left(y_{k}, K_{\varepsilon_{k}}\right)$. Then either

(i) $\exists\left[y_{\varepsilon}\right] \in \operatorname{ext}(K):\left[y_{\varepsilon}\right]$ joins points of the sequence $\left(y_{k}\right)_{k \in \mathbb{N}}$ at $\left(\varepsilon_{k}\right)_{k \in \mathbb{N}}$

or

(i) $\exists\left[\bar{y}_{\varepsilon}\right],\left[\bar{x}_{\varepsilon}\right]$ joining points of the sequences $\left(y_{k}\right)_{k \in \mathbb{N}}$ and $\left(x_{k}\right)_{k \in \mathbb{N}}$ (respectively) at $\left(\varepsilon_{k}\right)_{k \in \mathbb{N}}$ such that $\left[\bar{y}_{\varepsilon}\right]=\left[\bar{x}_{\varepsilon}\right]$ and $\bar{x}_{\varepsilon} \in K_{\varepsilon} \forall \varepsilon$.

Proof. We can always pick a point $e_{\varepsilon} \in K_{\varepsilon}^{c}$ so that $e:=\left[e_{\varepsilon}\right] \in \operatorname{ext}(K)$; in fact, since $\left(K_{\varepsilon}\right)$ is sharply bounded, we can find $e_{\varepsilon} \in \mathbb{R}^{n} \backslash K_{\varepsilon}$ so that $d\left(e_{\varepsilon}, K_{\varepsilon}\right)>1$ and $\left(e_{\varepsilon}\right)$ is moderate. We can also take a point $i_{\varepsilon} \in K_{\varepsilon}$ for each $\varepsilon$ because, without loss of generality, we can assume that $K_{\varepsilon} \neq \emptyset$ for all $\varepsilon$.

The first alternative in the statement is realized if

$$
\exists b \in \mathbb{R}_{>0} \exists N \in \mathbb{N} \forall k \geq N:\left|y_{k}-x_{k}\right|>\varepsilon_{k}^{b} .
$$

Set $y_{\varepsilon}:=y_{k}$ if $\varepsilon=\varepsilon_{k}$ and $y_{\varepsilon}:=e_{\varepsilon}$ otherwise. Then, if $\varepsilon=\varepsilon_{k}$, by (3.4) we have $d\left(y_{\varepsilon}, K_{\varepsilon}\right)=\left|y_{k}-x_{k}\right|>\varepsilon^{b} ;$ otherwise $d\left(y_{\varepsilon}, K_{\varepsilon}\right)=d\left(e_{\varepsilon}, K_{\varepsilon}\right)>1 \geq \varepsilon^{b}$. Therefore, $\left[y_{\varepsilon}\right] \in\left\langle K_{\varepsilon}^{c}\right\rangle=\operatorname{ext}(K)$ and, of course, $\left[y_{\varepsilon}\right]$ joins points of the sequence $\left(y_{k}\right)_{k \in \mathbb{N}}$ at $\left(\varepsilon_{k}\right)_{k \in \mathbb{N}}$.

Vice versa, if

$$
\forall h \in \mathbb{N} \exists k_{h}>h:\left|y_{k_{h}}-x_{k_{h}}\right| \leq \varepsilon_{k_{h}}^{h},
$$

then we can set $\bar{y}_{\varepsilon}:=y_{k_{h}}, \bar{x}_{\varepsilon}:=x_{k_{h}}$ if $\varepsilon=\varepsilon_{k_{h}}$ and $\bar{y}_{\varepsilon}:=\bar{x}_{\varepsilon}:=i_{\varepsilon}$ otherwise. Then, if $\varepsilon=\varepsilon_{k_{h}}$, by (3.5) we have $\left|\bar{y}_{\varepsilon}-\bar{x}_{\varepsilon}\right|=\left|y_{k_{h}}-x_{k_{h}}\right| \leq \varepsilon_{k_{h}}^{h}=\varepsilon^{h}$; otherwise $\left|\bar{y}_{\varepsilon}-\bar{x}_{\varepsilon}\right|=\left|i_{\varepsilon}-i_{\varepsilon}\right|=0$, and (i) follows.

We use the above result to guarantee that the maximum values of any partial derivative $\partial^{\alpha} f$ are attained on $K$ and not outside, as precisely stated in the following

Lemma 22. Let $\left(u_{\varepsilon}\right)$ and $K=\left[K_{\varepsilon}\right]$ satisfy Def. 19, then

$$
\forall \alpha \in \mathbb{N}^{n} \forall i=0, \ldots, n:\left[\sup _{y \in \mathbb{R}^{n}}\left|\partial^{\alpha} u_{\varepsilon}^{i}(y)\right|\right]=\left[\sup _{x \in K_{\varepsilon}}\left|\partial^{\alpha} u_{\varepsilon}^{i}(x)\right|\right] .
$$

Proof. By contradiction, assume that

$$
\left[\sup _{y \in \mathbb{R}^{n}}\left|\partial^{\alpha} u_{\varepsilon}^{i}(y)\right|\right] \neq\left[\sup _{x \in K_{\varepsilon}}\left|\partial^{\alpha} u_{\varepsilon}^{i}(x)\right|\right] .
$$

For simplicity of notation, set $v_{\varepsilon}:=\partial^{\alpha} u_{\varepsilon}^{i}$. Inequality (3.7) means

$$
\exists a \in \mathbb{R}_{>0} \exists \varepsilon_{k} \searrow 0 \forall k \in \mathbb{N}:\left|\sup _{y \in \mathbb{R}^{n}}\right| v_{\varepsilon_{k}}(y)\left|-\sup _{x \in K_{\varepsilon}}\right| v_{\varepsilon_{k}}(x)||>\varepsilon_{k}^{a} .
$$


Thus

$$
\forall k \in \mathbb{N} \exists y_{k} \in \mathbb{R}^{n}: \varepsilon_{k}^{a}+\sup _{x \in K_{\varepsilon_{k}}}\left|v_{\varepsilon_{k}}(x)\right|<\left|v_{\varepsilon_{k}}\left(y_{k}\right)\right| .
$$

We can hence apply Lemma 21. In the first case (i) we have $y:=\left[y_{\varepsilon}\right] \in \operatorname{ext}(K)$ so that $\left[v_{\varepsilon}\left(y_{\varepsilon}\right)\right]=0$ by Def. 19 (ii). Since $\left[y_{\varepsilon}\right]$ joins points of $\left(y_{k}\right)_{k \in \mathbb{N}}$ at $\left(\varepsilon_{k}\right)_{k \in \mathbb{N}}$, from (3.8) and $\left[v_{\varepsilon}\left(y_{\varepsilon}\right)\right]=0$ we get

$$
\varepsilon_{k}^{a} \leq \varepsilon_{k}^{a}+\sup _{x \in K_{\varepsilon_{k}}}\left|v_{\varepsilon_{k}}(x)\right|<\left|v_{\varepsilon_{k}}\left(y_{k}\right)\right|=\left|v_{\varepsilon_{k}}\left(y_{\varepsilon_{k}}\right)\right|<\varepsilon_{k}^{a+1},
$$

for $k$ sufficiently big, which gives a contradiction in the first case. In the second case, i.e., (i) of Lemma 21, we have $\bar{y}:=\left[\bar{y}_{\varepsilon}\right]=\bar{x}:=\left[\bar{x}_{\varepsilon}\right] \in K \subseteq U$. Therefore $\left[v_{\varepsilon}\left(\bar{y}_{\varepsilon}\right)\right]=\left[v_{\varepsilon}\left(\bar{x}_{\varepsilon}\right)\right]$ by the definition of GSF. Since $\left[\bar{y}_{\varepsilon}\right]$ and $\left[\bar{x}_{\varepsilon}\right]$ join points of $\left(y_{k}\right)_{k \in \mathbb{N}}$ and $\left(x_{k}\right)_{k \in \mathbb{N}}$, respectively, at $\left(\varepsilon_{k}\right)_{k \in \mathbb{N}}$, for $k$ sufficiently big we have

$$
\begin{aligned}
\varepsilon_{k}^{a}+\sup _{x \in K_{\varepsilon_{k}}}\left|v_{\varepsilon_{k}}(x)\right| & <\left|v_{\varepsilon_{k}}\left(y_{k}\right)\right| \leq\left|v_{\varepsilon_{k}}\left(y_{k}\right)-v_{\varepsilon_{k}}\left(x_{k}\right)\right|+\left|v_{\varepsilon_{k}}\left(x_{k}\right)\right| \\
& \leq \varepsilon_{k}^{a+1}+\sup _{x \in K_{\varepsilon_{k}}}\left|v_{\varepsilon_{k}}(x)\right|,
\end{aligned}
$$

leading to a contradiction also in the second case.

The previous result will be essential to prove that any compactly supported GSF can be extended to the whole of $\widetilde{\mathbb{R}}^{n}$, and to define an $\widetilde{\mathbb{R}}$-valued norm of $f$ that does not depend on $K$.

Using Lemma 22, we can prove that any derivative of a compactly supported GSF is globally bounded in an appropriate sense:

Lemma 23. Let the net $\left(u_{\varepsilon}\right)$ satisfies Def. 19, then

$$
\forall \alpha \in \mathbb{N}^{n} \exists C \in \widetilde{\mathbb{R}} \forall \beta \in \mathbb{N}^{n}:|\beta| \leq|\alpha| \Rightarrow\left[\sup _{y \in \mathbb{R}^{n}}\left|\partial^{\beta} u_{\varepsilon}(y)\right|\right] \leq C .
$$

Proof. By the extreme value property Prop. 2 we can set $C_{i \beta}:=\left[\sup _{y \in K_{\varepsilon}}\left|\partial^{\beta} u_{\varepsilon}^{i}(y)\right|\right]$, where $\beta \in \mathbb{N}^{n},|\beta| \leq|\alpha|$, and $i=1, \ldots, n$. Set $C:=1+\sqrt{n} \max _{\substack{|\beta| \leq|<| \\ 1<i<n}} C_{i \beta}$. Then $C>C_{i \beta}$ and property (3.6) yields $\left[\sup _{y \in \mathbb{R}^{n}}\left|\partial^{\beta} u_{\varepsilon}^{i}(y)\right|\right]=\left[\sup _{x \in K_{\varepsilon}}\left|\partial^{\beta} u_{\varepsilon}^{i}(x)\right|\right]=C_{i \beta}$ and hence $\left[\sup _{y \in \mathbb{R}^{n}}\left|\partial^{\beta} u_{\varepsilon}(y)\right|\right]<C$.

Moreover, compactly supported GSF can be extended in a unique way to the entire $\widetilde{\mathbb{R}}^{n}$ :

Theorem 24. Let $\emptyset \neq K \Subset_{f} U \subseteq \widetilde{\mathbb{R}}^{n}$ and $f \in \mathcal{G D}_{K}\left(U, \widetilde{\mathbb{R}}^{d}\right)$ be defined by $\left(u_{\varepsilon}\right)$ which satisfies Def. 19. Then $\left(u_{\varepsilon}\right)$ defines a GSF of the type $\widetilde{\mathbb{R}}^{n} \longrightarrow \widetilde{\mathbb{R}}^{d}$ and there exists one and only one $\bar{f} \in \mathcal{G} \mathcal{D}_{K}\left(\widetilde{\mathbb{R}}^{n}, \widetilde{\mathbb{R}}^{d}\right)$ such that:

(i) $\left.\bar{f}\right|_{K}=\left.f\right|_{K}$

(ii) $\left.\bar{f}\right|_{\operatorname{ext}(K)}=0$. 
Moreover, this $\bar{f}$ satisfies

(iii) If $U$ is sharply open and $\alpha \in \mathbb{N}^{n}$, then $\left.\partial^{\alpha} \bar{f}\right|_{U}=\partial^{\alpha} f$

(i) $\left.\bar{f}\right|_{\widetilde{\mathbb{R}}_{c}^{n}}$ can be identified with a Colombeau generalized function.

Proof. The existence part follows by showing that for all $\alpha \in \mathbb{N}^{n}$ and all $\left[x_{\varepsilon}\right] \in \widetilde{\mathbb{R}}^{n}$ we have $\left(\partial^{\alpha} u_{\varepsilon}\left(x_{\varepsilon}\right)\right) \in \mathbb{R}_{M}^{d}$. This follows since Lemma 23 yields that for any $\alpha \in \mathbb{N}^{n}$ there exists some $\left(C_{\alpha \varepsilon}\right) \in \mathbb{R}_{M}^{n}$ such that $\left|\sup _{x \in \mathbb{R}^{n}} \partial^{\alpha} u_{\varepsilon}(x)\right| \leq C_{\alpha \varepsilon}$ for all $\varepsilon$ small. To prove uniqueness, let $g \in \mathcal{G} \mathcal{D}_{K}\left(\widetilde{\mathbb{R}}^{n}, \widetilde{\mathbb{R}}^{d}\right)$ be such that $\left.g\right|_{K}=\left.\bar{f}\right|_{K}=f$ and $\left.g\right|_{\operatorname{ext}(K)}=$ $\left.\bar{f}\right|_{\operatorname{ext}(K)}=0$. By contradiction, assume that $\bar{f}(y)=\left[u_{\varepsilon}\left(y_{\varepsilon}\right)\right] \neq g(y)=\left[v_{\varepsilon}\left(y_{\varepsilon}\right)\right]$, for some $y=\left[y_{\varepsilon}\right] \in \widetilde{\mathbb{R}}^{n}$, where $\left(v_{\varepsilon}\right)$ defines $g$. Thus

$$
\exists a \in \mathbb{R}_{>0} \exists \varepsilon_{k} \searrow 0 \forall k \in \mathbb{N}:\left|u_{\varepsilon_{k}}\left(y_{\varepsilon_{k}}\right)-v_{\varepsilon_{k}}\left(y_{\varepsilon_{k}}\right)\right|>\varepsilon_{k}^{a} .
$$

By Lemma 21, this leaves two possibilities. In the first one, there exists a point $z=\left[z_{\varepsilon}\right] \in \operatorname{ext}(K)$ which joins points of the sequence $\left(y_{\varepsilon_{k}}\right)_{k \in \mathbb{N}}$ at $\left(\varepsilon_{k}\right)_{k \in \mathbb{N}}$. Therefore $g(z)=\left[v_{\varepsilon}\left(z_{\varepsilon}\right)\right]=\bar{f}(z)=\left[u_{\varepsilon}\left(z_{\varepsilon}\right)\right]=0$, which gives a contradiction at $\varepsilon=\varepsilon_{k}$ when compared with (3.9). In the second one, there exists a point $\bar{z}=\left[\bar{z}_{\varepsilon}\right] \in K$ joining points of the sequence $\left(y_{\varepsilon_{k}}\right)_{k \in \mathbb{N}}$ at $\left(\varepsilon_{k}\right)_{k \in \mathbb{N}}$. Once again, we have $g(\bar{z})=\left[v_{\varepsilon}\left(\bar{z}_{\varepsilon}\right)\right]=$ $\bar{f}(\bar{z})=\left[u_{\varepsilon}\left(\bar{z}_{\varepsilon}\right)\right]=f(z)$, in contradiction to (3.9).

Furthermore, [18, Thm. 31] implies claim (iii). Finally, (i) follows from the isomorphism $\mathcal{G C}^{\infty}\left(\widetilde{\mathbb{R}}_{c}, \widetilde{\mathbb{R}}^{d}\right) \simeq \mathcal{G}^{s}(\mathbb{R})^{d}$.

We also have this simple but useful result:

Lemma 25. Let $\emptyset \neq K \Subset_{f} U \subseteq \widetilde{\mathbb{R}}^{n}, U$ be a sharply open set, $f \in \mathcal{G D}_{K}\left(U, \widetilde{\mathbb{R}}^{d}\right)$ and $\alpha \in \mathbb{N}^{n}$. Then $\partial^{\alpha} f \in \mathcal{G} \mathcal{D}_{K}\left(U, \widetilde{\mathbb{R}}^{d}\right)$.

Thm. 24 opens the possibility to restrict our attention to compactly supported GSF whose domain is the entire $\widetilde{\mathbb{R}}^{n}$, as stated in the following

Theorem 26. For $\emptyset \neq K \Subset_{f} \widetilde{\mathbb{R}}^{n}$ and $Y \subseteq \widetilde{\mathbb{R}}^{d}$ set

$$
\mathcal{G D}^{\mathrm{g}}(K, Y):=\left\{f \in \mathcal{G C}^{\infty}\left(\widetilde{\mathbb{R}}^{n}, Y\right)|f|_{\operatorname{ext}(K)}=0\right\},
$$

where the g superscript means globally defined. Let $K \subseteq U \subseteq \widetilde{\mathbb{R}}^{n}$, then

(i) If $f \in \mathcal{G D}^{g}(K, Y)$, then $\left.f\right|_{U} \in \mathcal{G} \mathcal{D}_{K}(U, Y)$.

(ii) If $f \in \mathcal{G D}_{K}\left(U, \widetilde{\mathbb{R}}^{d}\right)$, then $\exists ! \bar{f} \in \mathcal{G} \mathcal{D}^{\mathrm{g}}\left(K, \widetilde{\mathbb{R}}^{d}\right):\left.\bar{f}\right|_{K}=\left.f\right|_{K}$.

(iii) $\mathcal{G D}^{\mathrm{g}}(K, Y)=\mathcal{G} \mathcal{D}_{K}\left(\widetilde{\mathbb{R}}^{n}, Y\right)$.

Proof. (i): Let $\left(u_{\varepsilon}\right)$ be any net that defines $f$, so that $u_{\varepsilon} \in \mathcal{C}^{\infty}\left(\mathbb{R}^{n}, \mathbb{R}^{d}\right)$. Clearly $\left.f\right|_{U} \in \mathcal{G C}^{\infty}(U, Y)$, so for all $\alpha \in \mathbb{N}^{n}$ and $x=\left[x_{\varepsilon}\right] \in \operatorname{ext}(K)$ it remains to show that $\left[\partial^{\alpha} u_{\varepsilon}\left(x_{\varepsilon}\right)\right]=0$. By Lem. 15, we get $\operatorname{ext}(K)=\left\langle K_{\varepsilon}^{c}\right\rangle$, which is a sharply open set. So $x \in \operatorname{ext}(K)$ yields $B_{r}(x) \subseteq \operatorname{ext}(K)$ for some $r \in \widetilde{\mathbb{R}}_{>0}$, and hence $\left.f\right|_{B_{r}(x)}=0$. Thereby $\partial^{\alpha} f(x)=\left[\partial^{\alpha} u_{\varepsilon}\left(x_{\varepsilon}\right)\right]=0$. 
(ii): This is exactly Thm. 24.

(iii): This follows directly from (i) by setting $U=\widetilde{\mathbb{R}}^{n}$, and by Def. 19 which yields $\left.f\right|_{\operatorname{ext}(K)}=0$.

For the extension of property (ii) to arbitrary codomains $Y \subseteq \widetilde{\mathbb{R}}^{d}$ (provided that $U$ is strongly internal) see Thm. 62 below.

Note explicitly that in (3.10), instead of the more technical properties of Def. 19, we have a concise and simpler pointwise condition. Notwithstanding this and several other positive aspects of definition (3.10) (see the second part of Lem. 17 and the following Thm. 27), in the present work, we prefer not to change Def. 19 in favor of (3.10): On the one hand, Def. 19 is nearer to the classical definition of $\mathcal{D}_{K}(\Omega)$, where the domain is $\Omega \subseteq \mathbb{R}^{n}$; on the other hand, for applications of these notions to geometry, locally defined functions are a more natural setting. We can also summarize these results by saying that compactly supported GSF have a good notion of being compactly supported, and globally defined compactly supported GSF are in addition well-behaved with respect to the concept of support introduced above. This is also clearly confirmed by the following

Theorem 27. Let $Y \subseteq \widetilde{\mathbb{R}}^{d}$, and $f \in \mathcal{G C} \mathcal{C}^{\infty}\left(\widetilde{\mathbb{R}}^{n}, Y\right)$ such that $\operatorname{supp}(f) \neq \emptyset$. Then $f \in \mathcal{G} \mathcal{D}_{K}\left(\widetilde{\mathbb{R}}^{n}, Y\right)$ if and only if $\operatorname{supp}(f) \subseteq K$.

Proof. One implication is immediate from Remark 20.(i). Conversely, if $\operatorname{supp}(f) \subseteq$ $K$, by Thm. 26.(iii) we have to show that $\left.f\right|_{\operatorname{ext}(K)}=0$. Let $x \in \operatorname{ext}(K)$ but assume that $f(x) \neq 0$. Pick any $y \in\left\{y^{\prime} \in \widetilde{\mathbb{R}}^{n}|| f\left(y^{\prime}\right) \mid>0\right\}$, which is non-empty because $\operatorname{supp}(f) \neq \emptyset$. Let $\left(u_{\varepsilon}\right)$ be a net that defines $f$ and let $y=\left[y_{\varepsilon}\right]$. Since $f(x) \neq 0$, there exists $S \subseteq I$ such that $e_{S} \neq 0$ and $\left|f(x) e_{S}\right|>0$. Then setting $z:=x e_{S}+y e_{S^{c}}$, we have $|f(z)|>0$ and hence $z \in \operatorname{supp}(f) \subseteq K$. But then $x e_{S}=z e_{S} \in K e_{S}$ which, by Lem. 14, yields $x \notin \operatorname{ext}(K)$, a contradiction.

3.2. Examples of compactly supported GSF. A first class of examples comes by considering each $\varphi \in \mathcal{D}_{K}(\Omega), K \Subset \Omega \subseteq \mathbb{R}^{n}$. Indeed, it suffices to set $K_{\varepsilon}:=K$ and $u_{\varepsilon}(x):=\varphi(x)$ if $x \in \Omega$ and $u_{\varepsilon}(x):=0$ otherwise to have that $\varphi \in \mathcal{G D} \widetilde{K}_{\widetilde{\Omega}}\left(\widetilde{\Omega}_{c}, \widetilde{\mathbb{R}}\right)$, where we recall that $\widetilde{K}=[K]$. Therefore $\mathcal{D}_{K}(\Omega) \subseteq \mathcal{G} \mathcal{D}_{\widetilde{K}}\left(\widetilde{\Omega}_{c}, \widetilde{\mathbb{R}}\right)$.

Moreover, since any given CGF can be defined by a net $\left(u_{\varepsilon}\right)$ of maps with sharply bounded compact supports, we have the following result:

Theorem 28. Let $\Omega$ be an open subset of $\mathbb{R}^{n}$ and $J=\left[J_{\varepsilon}\right] \in \widetilde{\mathbb{R}}$ be a CGN such that $\lim _{\varepsilon \rightarrow 0^{+}} J_{\varepsilon}=+\infty$. Set $K_{\varepsilon}:=\left\{x \in \Omega|| x \mid \leq J_{\varepsilon}\right\}$ and $K:=\left[K_{\varepsilon}\right]$. Then for all $f \in \mathcal{G C}^{\infty}\left(\widetilde{\Omega}_{c}, \widetilde{\mathbb{R}}^{d}\right)$ there exists $\bar{f} \in \mathcal{G} \mathcal{D}_{K}\left(\widetilde{\mathbb{R}}^{n}, \widetilde{\mathbb{R}}^{d}\right)$ such that $\left.\bar{f}\right|_{\widetilde{\Omega}_{c}}=f$.

Proof. Set $U_{\varepsilon}:=\left\{x \in \Omega|| x \mid<\frac{1}{2} J_{\varepsilon}\right\}$ so that $U_{\varepsilon} \subseteq K_{\varepsilon}$ for $\varepsilon$ small. Let $\chi_{\varepsilon} \in$ $\mathcal{C}^{\infty}(\Omega, \mathbb{R})$ be such that $\left.\chi\right|_{U_{\varepsilon}}=1$ and $\operatorname{supp}\left(\chi_{\varepsilon}\right) \subseteq K_{\varepsilon}$. Let $f \in \mathcal{G C}^{\infty}\left(\widetilde{\Omega}_{c}, \widetilde{\mathbb{R}}^{d}\right)$ be 
represented by $\left(v_{\varepsilon}\right)$, with $v_{\varepsilon} \in \mathcal{C}^{\infty}\left(\mathbb{R}^{n}, \mathbb{R}^{d}\right)$, and set $u_{\varepsilon}:=\chi_{\varepsilon} \cdot v_{\varepsilon}$. Then each $u_{\varepsilon}$ is compactly supported and any $x=\left[x_{\varepsilon}\right] \in \widetilde{\Omega}_{c}$ satisfies $x_{\varepsilon} \in U_{\varepsilon}$ for $\varepsilon$ small because $\lim _{\varepsilon \rightarrow 0^{+}} J_{\varepsilon}=+\infty$. Therefore $\bar{f}:=\left[u_{\varepsilon}(-)\right] \in \mathcal{G} \mathcal{D}_{K}\left(\widetilde{\mathbb{R}}^{n}, \widetilde{\mathbb{R}}^{d}\right)$, and if $x_{\varepsilon} \in U_{\varepsilon}$ then $u_{\varepsilon}\left(x_{\varepsilon}\right)=v_{\varepsilon}\left(x_{\varepsilon}\right)$, so $\left.\bar{f}\right|_{\widetilde{\Omega}_{c}}=f$.

This theorem gives an infinity of non-trivial examples of compactly supported GSF. Moreover, even though $\bar{f}$ depends on the fixed infinite number $J \in \widetilde{\mathbb{R}}$, every such $\bar{f}$ contains all the information of the original CGF $f$ because $\left.\bar{f}\right|_{\widetilde{\Omega}_{c}}=f$.

Finally, the constant function $f(x)=1$ for all $x \in \widetilde{\mathbb{R}}$ is not compactly supported. In fact, by contradiction, assume that $f$ admits $\left(u_{\varepsilon}\right)$ and $\left(K_{\varepsilon}\right)$ such that Def. 19 holds. Then choosing $r$ large enough that $\mathrm{d} \varepsilon^{-r} \in \widetilde{\mathbb{R}} \backslash K$ we arrive at $f\left(\mathrm{~d} \varepsilon^{-r}\right)=$ $\left[u_{\varepsilon}\left(\varepsilon^{-r}\right)\right]=0$.

\section{Generalized norms on $\mathcal{G} \mathcal{D}_{K}$ and $\mathcal{G D}$}

As a first step to topologizing the spaces $\mathcal{G} \mathcal{D}_{K}$ and $\mathcal{G D}$ we prove:

Theorem 29. Let $\emptyset \neq K \Subset_{f} U \subseteq \widetilde{\mathbb{R}}^{n}$, then

(i) $\mathcal{G D}_{K}\left(U, \widetilde{\mathbb{R}}^{d}\right)$ is an $\widetilde{\mathbb{R}}$-module

(ii) For all non-empty $H \Subset_{f} U$, the inclusion $K \subseteq H$ implies $\mathcal{G D}_{K}\left(U, \widetilde{\mathbb{R}}^{d}\right) \subseteq$ $\mathcal{G} \mathcal{D}_{H}\left(U, \widetilde{\mathbb{R}}^{d}\right)$.

Proof. (i) is immediate from Def. 19.

(ii): Take $f \in \mathcal{G D}_{K}\left(U, \widetilde{\mathbb{R}}^{d}\right)$. Since $K \subseteq H$, by [27, Prop. 2.8] for each representative $\left(K_{\varepsilon}\right)$ of $K$ we get the existence of a representative $\left(H_{\varepsilon}\right)$ of $H$ such that $K_{\varepsilon} \subseteq H_{\varepsilon}$ for all $\varepsilon$. Therefore, $\operatorname{ext}(K)=\left\langle K_{\varepsilon}^{c}\right\rangle \supseteq\left\langle H_{\varepsilon}^{c}\right\rangle=\operatorname{ext}(H)$ and hence the conclusion follows.

From the extreme value property, Prop. 2, it is natural to expect that the following CGN could serve as generalized $\widetilde{\mathbb{R}}$-valued norms.

Definition 30. Let $\emptyset \neq K \Subset_{\mathrm{f}} U \subseteq \widetilde{\mathbb{R}}^{n}$, where $U$ is a sharply open set. Let $m \in \mathbb{N}$ and $f \in \mathcal{G D}_{K}\left(U, \widetilde{\mathbb{R}}^{d}\right)$. Then

$$
\|f\|_{m, K}:=\max _{\substack{|\alpha| \leq m \\ 1 \leq i \leq d}} \max \left(\left|\partial^{\alpha} f^{i}\left(M_{\alpha i}\right)\right|,\left|\partial^{\alpha} f^{i}\left(m_{\alpha i}\right)\right|\right) \in \widetilde{\mathbb{R}},
$$

where $m_{\alpha i}, M_{\alpha i} \in K$ satisfy

$$
\forall x \in K: \partial^{\alpha} f^{i}\left(m_{\alpha i}\right) \leq \partial^{\alpha} f^{i}(x) \leq \partial^{\alpha} f^{i}\left(M_{\alpha i}\right) .
$$

The following result permits to calculate the (generalized) norm $\|f\|_{m, K}$ using any net $\left(v_{\varepsilon}\right)$ that defines $f$. In case the net $\left(v_{\varepsilon}\right)$ satisfies Def. 19 , it also permits to prove that this norm does not depend on $K$, as is the case for any ordinary compactly supported smooth function. 
Even though $\|f\|_{m, K} \in \widetilde{\mathbb{R}}$, using an innocuous abuse of language, in the following we will simply call $\|f\|_{m, K}$ a norm.

Proposition 31. Under the assumptions of Def. 30, let the set $K=\left[K_{\varepsilon}\right] \Subset_{f} \widetilde{\mathbb{R}}^{n}$. Then we have:

(i) If the net $\left(v_{\varepsilon}\right)$ defines $f$, then $\|f\|_{m, K}=\left[\max _{\substack{|\alpha| \leq m \\ 1 \leq i \leq d}} \sup _{x \in K_{\varepsilon}}\left|\partial^{\alpha} v_{\varepsilon}^{i}(x)\right|\right]$

(ii) If $\left(u_{\varepsilon}\right)$ defines $f$ and $\left(u_{\varepsilon}\right)$ satisfies Def. 19, then

$$
\|f\|_{m, K}=\left[\max _{\substack{|\alpha| \leq m \\ 1 \leq i \leq d}} \sup _{x \in \mathbb{R}^{n}}\left|\partial^{\alpha} u_{\varepsilon}^{i}(x)\right|\right] .
$$

Proof. In proving (i) we will also prove that the norm $\|f\|_{m, K}$ is well-defined, i.e. it does not depend on the particular choice of points $m_{\alpha i}, M_{\alpha i}$ as in Def. 30. As in the proof of Prop. 2, we get the existence of $\bar{m}_{\alpha i \varepsilon}, \bar{M}_{\alpha i \varepsilon} \in K_{\varepsilon}$ such that

$$
\forall x \in K_{\varepsilon}: \partial^{\alpha} v_{\varepsilon}^{i}\left(\bar{m}_{\alpha i \varepsilon}\right) \leq \partial^{\alpha} v_{\varepsilon}^{i}(x) \leq \partial^{\alpha} v_{\varepsilon}^{i}\left(\bar{M}_{\alpha i \varepsilon}\right) .
$$

Hence $\left|\partial^{\alpha} v_{\varepsilon}^{i}(x)\right| \leq \max \left(\left|\partial^{\alpha} v_{\varepsilon}^{i}\left(\bar{m}_{\alpha i \varepsilon}\right)\right|,\left|\partial^{\alpha} v_{\varepsilon}^{i}\left(\bar{M}_{\alpha i \varepsilon}\right)\right|\right)$. Thus

$$
\max _{\substack{|\alpha| \leq m \\ 1 \leq i \leq d}} \sup _{x \in K_{\varepsilon}}\left|\partial^{\alpha} v_{\varepsilon}^{i}(x)\right| \leq \max _{\substack{|\alpha| \leq m \\ 1 \leq i \leq d}} \max \left(\left|\partial^{\alpha} v_{\varepsilon}^{i}\left(\bar{m}_{\alpha i \varepsilon}\right)\right|,\left|\partial^{\alpha} v_{\varepsilon}^{i}\left(\bar{M}_{\alpha i \varepsilon}\right)\right|\right)
$$

But $\bar{m}_{\alpha i \varepsilon}, \bar{M}_{\alpha i \varepsilon} \in K_{\varepsilon}$, so

$$
\begin{aligned}
{\left[\max _{\substack{|\alpha| \leq m \\
1 \leq i \leq d}} \sup _{x \in K_{\varepsilon}}\left|\partial^{\alpha} v_{\varepsilon}^{i}(x)\right|\right] } & =\left[\max _{\substack{|\alpha| \leq m \\
1 \leq i \leq d}} \max \left(\left|\partial^{\alpha} v_{\varepsilon}^{i}\left(\bar{m}_{\alpha i \varepsilon}\right)\right|,\left|\partial^{\alpha} v_{\varepsilon}^{i}\left(\bar{M}_{\alpha i \varepsilon}\right)\right|\right)\right]= \\
& =\max _{\substack{|\alpha| \leq m \\
1 \leq i \leq d}} \max \left(\left|\partial^{\alpha} f^{i}\left(\bar{M}_{\alpha i}\right)\right|,\left|\partial^{\alpha} f^{i}\left(\bar{m}_{\alpha i}\right)\right|\right) .
\end{aligned}
$$

From this, both the fact that the norm $\|f\|_{m, K}$ is well-defined and claim (i) follow.

(ii): By Lemma 22, we have that

$$
\begin{aligned}
{\left[\max _{\substack{|\alpha| \leq m \\
1 \leq i \leq d}} \sup _{x \in K_{\varepsilon}}\left|\partial^{\alpha} u_{\varepsilon}^{i}(x)\right|\right] } & =\max _{\substack{|\alpha| \leq m \\
1 \leq i \leq d}}\left[\sup _{x \in K_{\varepsilon}}\left|\partial^{\alpha} u_{\varepsilon}^{i}(x)\right|\right]= \\
& =\max _{\substack{|\alpha| \leq m \\
1 \leq i \leq d}}\left[\sup _{x \in \mathbb{R}^{n}}\left|\partial^{\alpha} u_{\varepsilon}^{i}(x)\right|\right]=\left[\max _{\substack{|\alpha| \leq m \\
1 \leq i \leq d}} \sup _{x \in \mathbb{R}^{n}}\left|\partial^{\alpha} u_{\varepsilon}^{i}(x)\right|\right] .
\end{aligned}
$$

Corollary 32. Let $\emptyset \neq K \Subset_{f} U \subseteq \widetilde{\mathbb{R}}^{n}$, where $U$ is a sharply open set. Let $\emptyset \neq H \Subset_{f}$ $U$ and $m \in \mathbb{N}$. If $f \in \mathcal{G} \mathcal{D}_{K}\left(U, \widetilde{\mathbb{R}}^{d}\right) \cap \mathcal{G} \mathcal{D}_{H}\left(U, \widetilde{\mathbb{R}}^{d}\right)$, then $\|f\|_{m, K}=\|f\|_{m, H}=:\|f\|_{m}$.

Proof. The right hand side of (4.1) does not depend on $K$.

Another consequence of Prop. 31 is the following: 
Corollary 33. Let $U \subseteq \widetilde{\mathbb{R}}^{n}, f \in \mathcal{G D}\left(U, \widetilde{\mathbb{R}}^{d}\right)$ and $\bar{f} \in \mathcal{G D}\left(\widetilde{\mathbb{R}}^{n}, \widetilde{\mathbb{R}}^{d}\right)$ be the extension of $f$ defined in Thm. 24. Then for all $m \in \mathbb{N},\|f\|_{m}=\|\bar{f}\|_{m}$.

Our use of the term "norm" is justified by the following

Proposition 34. Let $\emptyset \neq K \Subset_{f} U \subseteq \widetilde{\mathbb{R}}^{n}$, where $U$ is a sharply open set. Let $f$, $g \in \mathcal{G D}_{K}\left(U, \widetilde{\mathbb{R}}^{d}\right)$ and $m \in \mathbb{N}$. Then

(i) $\|f\|_{m} \geq 0$

(ii) $\|f\|_{m}=0$ if and only if $f=0$

(iii) $\forall c \in \widetilde{\mathbb{R}}:\|c \cdot f\|_{m}=|c| \cdot\|f\|_{m}$

(iv) $\|f+g\|_{m} \leq\|f\|_{m}+\|g\|_{m}$.

(v) $\|f \cdot g\|_{m} \leq c_{m} \cdot\|f\|_{m} \cdot\|g\|_{m}$ for some $c_{m} \in \mathbb{R}_{>0}$.

Proof. (i), (iii) and (iv) follow directly from Prop. 31, as does (v), using the Leibniz rule. The 'if'-part of property (ii) follows from (4.1).

We now prove that also the space $\mathcal{G D}\left(U, \widetilde{\mathbb{R}}^{d}\right)$ is an $\widetilde{\mathbb{R}}$-module, at least for certain $U$ :

Proposition 35. Let $U \subseteq \widetilde{\mathbb{R}}^{n}$ be a non empty sharply open set. Assume that

$$
\forall K, H \Subset_{f} U: \operatorname{interl}(H \cup K) \subseteq U .
$$

Then $\mathcal{G D}\left(U, \widetilde{\mathbb{R}}^{d}\right)$ is an $\widetilde{\mathbb{R}}$-module.

Proof. Since in Thm. 29 we already proved that $\mathcal{G} \mathcal{D}_{K}\left(U, \widetilde{\mathbb{R}}^{d}\right)$ is closed with respect to products by scalars, we only need to prove that $\mathcal{G D}\left(U, \widetilde{\mathbb{R}}^{d}\right)$ is closed with respect to sum. Let $f \in \mathcal{G D}_{K}\left(U, \widetilde{\mathbb{R}}^{d}\right), g \in \mathcal{G D}_{H}\left(U, \widetilde{\mathbb{R}}^{d}\right)$ and let $\left(u_{\varepsilon}\right),\left(v_{\varepsilon}\right)$ satisfy Def. 19 for $f$ and $g$, respectively. Lemma 8 and [27, Prop. 2.8] imply that interl $(H \cup K)=$ $\left[H_{\varepsilon} \cup K_{\varepsilon}\right] \Subset_{\mathrm{f}} \widetilde{\mathbb{R}}^{n}$. But $\operatorname{ext}(H \cup K)=\left\langle H_{\varepsilon}^{c} \cap K_{\varepsilon}^{c}\right\rangle=\left\langle H_{\varepsilon}^{c}\right\rangle \cap\left\langle K_{\varepsilon}^{c}\right\rangle=\operatorname{ext}(H) \cap$ $\operatorname{ext}(K)$. Therefore, $\partial^{\alpha}\left(u_{\varepsilon}+v_{\varepsilon}\right)$ is zero on $\operatorname{ext}(H \cup K)$. By our assumption (4.2) $\emptyset \neq \operatorname{interl}(H \cup K) \Subset_{\mathrm{f}} U$, so that $f+g \in \mathcal{G D}\left(U, \widetilde{\mathbb{R}}^{d}\right)$.

In the following result, we give two general sufficient conditions for (4.2) to hold.

Proposition 36. Let $U \subseteq \widetilde{\mathbb{R}}^{n}$ be a non-empty sharply open set. If $U$ is $\widetilde{\mathbb{R}}$-convex or $U$ is a strongly internal set, then (4.2) holds.

Proof. Assume that $U$ is $\widetilde{\mathbb{R}}$-convex, i.e. $x h+(1-x) k \in U$ for all $h, k \in U$ and all $x \in[0,1]$. Then for all $H, K \subseteq U$ (even if we do not assume them to be functionally compact), and all $y \in \operatorname{interl}(H \cup K)$, we can write $y=e_{S} \cdot h+e_{S^{c}} \cdot k$ for some $S \subseteq I$ and $h \in H, k \in K$. Thus $y=e_{S} \cdot h+\left(1-e_{S}\right) \cdot k \in U$ since $e_{S} \in[0,1]$ and $h, k \in U$.

Now, assume that $U$ is a strongly internal set, i.e. for some net $\left(U_{\varepsilon}\right)$ of subsets of $\mathbb{R}^{n}$, we have $U=\left\langle U_{\varepsilon}\right\rangle$. We continue to use the notations for $y$ as above. Since $h, k \in U,\left[18\right.$, Thm. 8] entails that $d\left(h_{\varepsilon}, U_{\varepsilon}^{c}\right), d\left(k_{\varepsilon}, U_{\varepsilon}^{c}\right)>\varepsilon^{q}$ for some $q \in \mathbb{R}_{>0}$ and 
$\varepsilon$ small, where $h=\left[h_{\varepsilon}\right], k=\left[k_{\varepsilon}\right]$. But $y=e_{S} \cdot h+e_{S^{c}} \cdot k$, so for all $\varepsilon$ small, if $\varepsilon \in S$ then $y_{\varepsilon}=h_{\varepsilon}$ and if $\varepsilon \notin S$ then $y_{\varepsilon}=k_{\varepsilon}$. In any case, $d\left(y_{\varepsilon}, U_{\varepsilon}^{c}\right)>\varepsilon^{q}$, hence $y \in\left\langle U_{\varepsilon}\right\rangle=U$.

Example 37. If $U=(-1,1) \cup(2,4) \subseteq \widetilde{\mathbb{R}}$, then $U$ is a sharply open set, but it does not satisfy condition (4.2) of Prop. (35): let $H:=\left[\left[-\frac{1}{2}, \frac{1}{2}\right]_{\mathbb{R}}\right], K:=\left[\left[\frac{5}{2}, \frac{7}{2}\right]_{\mathbb{R}}\right]$ and $x_{\varepsilon}:=0$ if $\varepsilon \in\left[\frac{1}{n}, \frac{1}{n+1}\right)$ if $n \in \mathbb{N}_{>0}$ is even, and $x_{\varepsilon}:=3$ otherwise. Then $x:=\left[x_{\varepsilon}\right] \in \operatorname{interl}(H \cup K)$ but $x \notin U$. Moreover, let $\varphi \in \mathcal{D}_{\left[-\frac{1}{2}, \frac{1}{2}\right]_{\mathbb{R}}}(\mathbb{R}) \subseteq \mathcal{G} \mathcal{D}_{H}\left(\widetilde{\mathbb{R}}_{c}\right)$, $\psi \in \mathcal{D}_{\left[\frac{5}{2}, \frac{7}{2}\right]_{\mathbb{R}}}(\mathbb{R}) \subseteq \mathcal{G D}_{K}\left(\widetilde{\mathbb{R}}_{c}\right)$ be positive non-trivial functions. Then, as we showed in the proof of Prop. 35, the GSF $\varphi+\psi \in \mathcal{G C}^{\infty}(U, \widetilde{\mathbb{R}})$ is compactly supported in $\left[\left[-\frac{1}{2}, \frac{1}{2}\right]_{\mathbb{R}} \cup\left[\frac{5}{2}, \frac{7}{2}\right]_{\mathbb{R}}\right]=\operatorname{interl}(H \cup K) \nsubseteq U$. Finally, let $f:=\left.\varphi\right|_{U}$ and $g:=\left.\psi\right|_{U}$, so that $f \in \mathcal{G D}_{H}(U, \widetilde{\mathbb{R}})$ and $g \in \mathcal{G} \mathcal{D}_{K}(U, \widetilde{\mathbb{R}})$. Rem. 20.(i) yields that $f+g \notin \mathcal{G} \mathcal{D}_{J}(U, \widetilde{\mathbb{R}})$ for all $J \Subset_{\mathrm{f}} U$ : Otherwise, taking suitable sub-intervals $L$ of $\left(-\frac{1}{2}, \frac{1}{2}\right)_{\mathbb{R}}$ and $M$ of $\left(\frac{5}{2}, \frac{7}{2}\right)_{\mathbb{R}}$ where $f$ resp. $g$ do not vanish, we would have $[L \cup M] \subseteq \operatorname{supp}(f+g) \subseteq J \subseteq U$ (here $\operatorname{supp}(f+g)$ is the support as in Def. 11). But the inclusion $[L \cup M] \subseteq U$ is impossible - a counterexample can be constructed similar to the above $x$.

This example shows that an assumption like (4.2) is necessary to have the closure of the space $\mathcal{G D}(U, \widetilde{\mathbb{R}})$ with respect to sum.

\section{TOPOlOGical StRUCTURE ON $\mathcal{G} \mathcal{D}_{K}$}

Using our $\widetilde{\mathbb{R}}$-valued norms, it is now natural to define

Definition 38. Let $\emptyset \neq K \Subset_{\mathrm{f}} U \subseteq \widetilde{\mathbb{R}}^{n}$, where $U$ is a sharply open set. Let $f \in \mathcal{G D}_{K}\left(U, \widetilde{\mathbb{R}}^{d}\right), m \in \mathbb{N}, \rho \in \widetilde{\mathbb{R}}_{>0}$, then

(i) $\quad B_{\rho}^{m}(f):=\left\{g \in \mathcal{G} \mathcal{D}_{K}\left(U, \widetilde{\mathbb{R}}^{d}\right) \mid\|f-g\|_{m}<\rho\right\}$. In case any confusion could arise, we will use the more precise symbol $B_{\rho}^{m}(f, K):=B_{\rho}^{m}(f)$.

(ii) If $V \subseteq \mathcal{G D}_{K}\left(U, \widetilde{\mathbb{R}}^{d}\right)$, then we say that $V$ is a sharply open set if

$$
\forall v \in V \exists m \in \mathbb{N} \exists \rho \in \widetilde{\mathbb{R}}_{>0}: B_{\rho}^{m}(v) \subseteq V .
$$

Moreover, we say that $V$ is Fermat open if

$$
\forall v \in V \exists m \in \mathbb{N} \exists r \in \mathbb{R}_{>0}: B_{r}^{m}(v) \subseteq V .
$$

As in [18, Thm. 2] it follows that sharply open sets as well as Fermat open sets form topologies on $\mathcal{G} \mathcal{D}_{K}\left(U, \widetilde{\mathbb{R}}^{d}\right)$.

On the other hand, it is also natural to view the space $\mathcal{G D}_{K}\left(U, \widetilde{\mathbb{R}}^{d}\right)$ inside Garetto's theory $[11,12]$ of $\widetilde{\mathbb{R}}$-locally convex algebras. In this section, we will realize this 
comparison, proving that the space $\mathcal{G D}_{K}(U, \widetilde{\mathbb{R}})$ is a Fréchet $\widetilde{\mathbb{R}}$-module and a topological algebra. For this purpose, we will only consider the sharp topology. Indeed, as we will see below, the Fermat topology is less interesting in this context since it doesn't permit to prove the continuity of the product by scalars $(r, f) \in \widetilde{\mathbb{R}} \times \mathcal{G} \mathcal{D}_{K}\left(U, \widetilde{\mathbb{R}}^{d}\right) \mapsto r \cdot f \in \mathcal{G} \mathcal{D}_{K}\left(U, \widetilde{\mathbb{R}}^{d}\right)$.

In the following, we will always assume that $\emptyset \neq K \Subset_{\mathrm{f}} U \subseteq \widetilde{\mathbb{R}}^{n}$, where $U$ is a nonempty sharply open set. The main problem in performing this comparison, which doesn't permit to view our space $\mathcal{G D}_{K}(U, \widetilde{\mathbb{R}})$ as a particular case of the theory developed in $[11,12]$, is that the domain $U$ contains generalized points.

Using the valuation $v$ on $\widetilde{\mathbb{R}}$, it is natural to introduce the following notions:

Definition 39. Let $m \in \mathbb{N}$ and $f \in \mathcal{G D}(U, \widetilde{\mathbb{R}})$, then:

(i) $\quad v_{m}(f):=v\left(\|f\|_{m}\right) \in \mathbb{R}$

(ii) $\mathcal{P}_{m}(f):=e^{-v_{m}(f)}$.

From the properties of the valuation $v$ and of the e-norm $|-|_{e}=e^{-v(-)}$ on $\widetilde{\mathbb{R}}$ (see [4]), the following result directly follows.

Proposition 40. For each $m \in \mathbb{N}$, we have:

$$
\begin{aligned}
& v_{m}: \mathcal{G D}(U) \longrightarrow \mathbb{R} \cup\{+\infty\} \text { is a valuation, i.e. for all } f, g \in \mathcal{G} \mathcal{D}(U) \text { : } \\
& \text { - } v_{m}(0)=+\infty \\
& \text { - } v_{m}(\lambda \cdot f) \geq v(\lambda)+v_{m}(f) \quad \forall \lambda \in \widetilde{\mathbb{R}} \\
& \text { - } v_{m}\left(\mathrm{~d} \varepsilon^{a} \cdot f\right)=v\left(\mathrm{~d} \varepsilon^{a}\right)+v_{m}(f)=a+v_{m}(f) \quad \forall a \in \mathbb{R} \\
& \text { - } v_{m}(f+g) \geq \min \left[v_{m}(f), v_{m}(g)\right] \text {. } \\
& \text { (ii) } \mathcal{P}_{m}: \mathcal{G D}(U) \longrightarrow \mathbb{R} \text { is an ultra-pseudo-norm, i.e. for all } f, g \in \mathcal{G} \mathcal{D}(U) \text { : } \\
& \text { - } \mathcal{P}_{m}(f)=0 \text { if and only if } f=0 \\
& \text { - } \mathcal{P}_{m}(\lambda \cdot f) \leq|\lambda|_{e} \cdot \mathcal{P}_{m}(f) \quad \forall \lambda \in \widetilde{\mathbb{R}} \\
& \text { - } \mathcal{P}_{m}\left(\mathrm{~d} \varepsilon^{a} \cdot f\right)=\left|\mathrm{d} \varepsilon^{a}\right|_{e} \cdot \mathcal{P}_{m}(f)=e^{-a} \cdot \mathcal{P}_{m}(f) \quad \forall a \in \mathbb{R} \\
& \text { - } \mathcal{P}_{m}(f+g) \leq \max \left[\mathcal{P}_{m}(f), \mathcal{P}_{m}(g)\right] \text {. }
\end{aligned}
$$

The following result states that to define the sharp topology, instead of the above ultra-pseudo-norms we can equivalently use the countable family of generalized norms $\left(\|f\|_{m}\right)_{m \in \mathbb{N}}$.

\section{Theorem 41.}

(i) Sum and product in $\mathcal{G D}_{K}(U)$ are continuous in the sharp topology. Therefore, $\mathcal{G D}_{K}(U)$ is a topological $\widetilde{\mathbb{R}}$-algebra.

(ii) The product in $\mathcal{G D}_{K}(U)$ is continuous in the Fermat topology only on the subspace $\left\{(f, g) \in \mathcal{G D}_{K}(U) \times \mathcal{G} \mathcal{D}_{K}(U) \mid \forall m \in \mathbb{N}:\|f\|_{m},\|g\|_{m}<\infty\right\}$.

(iii) For $f \in \mathcal{G D}_{K}(U)$, set

$$
C_{r}^{m}(f):=\left\{g \in \mathcal{G D}_{K}(U) \mid \mathcal{P}_{m}(f-g)<r\right\} \quad\left(r \in \mathbb{R}_{>0}, m \in \mathbb{N}\right) .
$$


Then for each $q, s \in \mathbb{R}_{>0}$ we have:

(i) If $q \leq-\log r$, then $C_{r}^{m}(f) \subseteq B_{\mathrm{d} \varepsilon^{q}}^{m}(f)$

(ii) If $q \geq-\log s$ and $s<r$, then $B_{\mathrm{d} \varepsilon^{q}}^{m}(f) \subseteq C_{r}^{m}(f)$.

(iv) The sharp topology on $\mathcal{G D}_{K}(U)$ is the coarsest topology such that each $\mathcal{P}_{m}$ is continuous.

(v) $\mathcal{G D}_{K}(U)$ is a separated locally convex topological $\widetilde{\mathbb{R}}-$ module.

Proof. (i), (ii): Continuity of the sum in the sharp (and in the Fermat) topology follows directly from the triangle inequality Prop. 34.(iv). The continuity of the product at $\left(f_{0}, g_{0}\right)$ and property (ii) follow from Prop. 34 (v) via

$$
\begin{aligned}
\left\|f \cdot g-f_{0} \cdot g_{0}\right\|_{m} \leq & c_{m}\left(\left\|f-f_{0}\right\|_{m} \cdot\left\|g-g_{0}\right\|_{m}+\left\|f-f_{0}\right\|_{m} \cdot\left\|g_{0}\right\|_{m}+\right. \\
& \left.+\left\|f_{0}\right\|_{m} \cdot\left\|g-g_{0}\right\|_{m}\right) .
\end{aligned}
$$

(iii): Let us first assume $q \leq-\log r$ and $g \in C_{r}^{m}(f)$, so that $\mathcal{P}_{m}(f-g)<r$ and $v_{m}(f-g)>-\log r$. This implies

$$
\exists b>-\log r: \max _{\substack{|\alpha| \leq m \\ i \leq d}} \sup _{x \in K_{\varepsilon}}\left|\partial^{\alpha} u_{\varepsilon}^{i}(x)-\partial^{\alpha} v_{\varepsilon}^{i}(x)\right|=O\left(\varepsilon^{b}\right),
$$

where $\left(u_{\varepsilon}\right)$ and $\left(v_{\varepsilon}\right)$ define $f$ and $g$, respectively. Property (5.1) yields the existence of some $M>0$ such that for $\varepsilon$ sufficiently small we obtain

$$
\max _{\substack{|\alpha| \leq m \\ i \leq d}} \sup _{x \in K_{\varepsilon}}\left|\partial^{\alpha} u_{\varepsilon}^{i}(x)-\partial^{\alpha} v_{\varepsilon}^{i}(x)\right|+\varepsilon^{b} \leq(M+1) \cdot \varepsilon^{b}<\varepsilon^{-\log r} \leq \varepsilon^{q} .
$$

Therefore $\|f-g\|_{m}+\mathrm{d} \varepsilon^{b} \leq \mathrm{d} \varepsilon^{q}$, so $\|f-g\|_{m}<\mathrm{d} \varepsilon^{q}$.

Now, let us assume $q \geq-\log s, s<r$, and $g \in B_{\mathrm{d} \varepsilon^{q}}^{m}(f)$, so that $\|f-g\|_{m}<\mathrm{d} \varepsilon^{q}$. Therefore,

$$
\forall^{0} \varepsilon: \max _{\substack{|\alpha| \leq m \\ i \leq d}} \sup _{x \in K_{\varepsilon}}\left|\partial^{\alpha} u_{\varepsilon}^{i}(x)-\partial^{\alpha} v_{\varepsilon}^{i}(x)\right|<\varepsilon^{q} \leq \varepsilon^{-\log s} .
$$

Taking the $|-| e^{- \text {-norm we get }}$

$$
\left|\max _{|\alpha| \leq m} \sup _{\substack{i \leq d \\ x \in K_{\varepsilon}}}\right| \partial^{\alpha} u_{\varepsilon}^{i}(x)-\partial^{\alpha} v_{\varepsilon}^{i}(x)||_{e} \leq e^{\log s}=s<r,
$$

that is $\mathcal{P}_{m}(f-g)<r$ as claimed.

(iv) follows directly from (iii) and Prop. 40.(ii).

(v) follows from Prop. 34.(ii) and [11, Prop. 1.11].

5.1. Generalized functions and non-Archimedean properties. In this section, we want to clarify some relationships between the classical notion of convexity, the notion of $\widetilde{\mathbb{R}}$-convexity of [11] and the use of $\widetilde{\mathbb{R}}$-valued norms.

We have seen that balls $B_{\rho}^{m}(0), \rho \in \widetilde{\mathbb{R}}_{>0}$, define a neighborhood system of 0 for $\mathcal{G D}_{K}(U)$; they are convex in the usual sense, i.e. if $f, g \in B_{\rho}^{m}(0), t \in[0,1]$ (in 
particular if $\left.t \in[0,1]_{\mathbb{R}}\right)$, then

$$
\|t f+(1-t) g\|_{m} \leq t\|f\|_{m}+(1-t)\|g\|_{m}<t \rho+(1-t) \rho=\rho .
$$

Moreover, each ball $B_{\rho}^{m}(0)$ is also balanced: if $t \in \widetilde{\mathbb{R}},|t| \leq 1$, then $t \cdot B_{\rho}^{m}(0) \subseteq B_{\rho}^{m}(0)$. However, this space is not a classical locally convex topological vector space over the field $\mathbb{R}$ because of two reasons: (i) the product by scalars is not continuous with respect to the Euclidean topology on $\mathbb{R}$, (ii) Lemma 23 implies that the property

$$
\forall f \in \mathcal{G D}_{K}(U) \exists t \in \widetilde{\mathbb{R}}: \quad f \in t \cdot B_{\rho}^{m}(0)
$$

holds for $t \in \widetilde{\mathbb{R}}$ but it cannot be extended to $t \in \mathbb{R}$. As we have seen in the proof of Thm. 41 (ii), this is a necessary consequence of the existence of generalized functions with infinite $\widetilde{\mathbb{R}}$-valued $\|-\|_{m}$-norm.

On the other hand, even though the sets $C_{r}^{m}(0)$ are defined using $\mathbb{R}$ only, i.e. without mentioning any non-Archimedean property, they satisfy

$$
\forall f \in C_{r}^{m}(0) \forall \lambda \in \mathbb{R}: \lambda \cdot f \in C_{r}^{m}(0),
$$

and this is possible only because they are infinitesimal sets. In fact, we have seen in Thm. 41 that $C_{r}^{m}(0) \subseteq B_{\mathrm{d} \varepsilon^{q}}^{m}(0)$ for $q \leq-\log r$.

More generally, a set $A \subseteq \mathcal{G D}_{K}(U)$ can be $\widetilde{\mathbb{R}}$-balanced (see [11]), i.e.

$$
\lambda A \subseteq A \quad \forall \lambda \in \widetilde{\mathbb{R}}:|\lambda|_{e} \leq 1,
$$

and at the same time can be thought of as "small" only in case $A$ consists infinitesimal points. For example, the ball $B_{\mathrm{d} \varepsilon^{b}}^{m}(0)$ is $\widetilde{\mathbb{R}}$-balanced, but $B_{1}^{m}(0)$ is not. In fact, we have

Lemma 42. Suppose that $A \subseteq \mathcal{G D}_{K}(U)$ and $m \in \mathbb{N}$ are such that

$$
A+A \subseteq A
$$

$$
\exists r \in \mathbb{R}_{>0}: A \subseteq B_{r}^{m}(0) .
$$

Then every element $u \in A$ has infinitesimal norm: $\|u\|_{m} \approx 0$.

Proof. In fact, (5.3) implies $n \cdot u \in A \subseteq B_{r}^{m}(0)$ for all $n \in \mathbb{N}_{\neq 0}$. Therefore, $\|u\|_{m}<\frac{r}{n}$ for all $n \in \mathbb{N}_{\neq 0}$, which proves our claim.

Let us note that condition (5.3) holds both for $A$ which is $\widetilde{\mathbb{R}}$-balanced or $\widetilde{\mathbb{R}}$ convex.

These remarks permit to show that in dealing with generalized functions, we are naturally induced to consider a topology on $\widetilde{\mathbb{R}}$ which contains infinitesimal neighborhoods (hence inducing the discrete topology on $\mathbb{R}$, see [17]). This is due to the coexistence of a continuous product by scalars and of an infinite element in $\mathcal{G D}_{K}(U)$, as stated in the following general result. In a possible interpretation of 
its statement, we can think of $R$ as $\mathbb{R}$ with a topology $\tau, \widetilde{R}$ as $\widetilde{\mathbb{R}}$ with the sharp topology $\tilde{\tau}$, and $<_{\widetilde{R}}$ as the strict order relation $<$ of Lemma 1 .

Theorem 43. Let $\left(R,+_{R}, \cdot_{R},<_{R}, \tau\right)$ and $\left(\widetilde{R},+_{\widetilde{R}}, \cdot_{\tilde{R}},<_{\widetilde{R}}, \tilde{\tau}\right)$ be Hausdorff topological ordered rings such that $(\mathbb{R},+, \cdot,<)$ is a substructure of $\left(R,+_{R}, \cdot_{R},<_{R}\right)$, which in turn is a substructure of $\left(\widetilde{R},+\widetilde{R}, \cdot \widetilde{R},<_{\tilde{R}}\right)$ and such that

$$
\forall r \in R \forall s \in \mathbb{R}: r<_{\widetilde{R}} s \Longrightarrow r<_{R} s .
$$

Let $\left(G,+_{G}, \cdot_{G}, \sigma\right)$ be a Hausdorff topological $R$-module, and $|-|_{G}: G \longrightarrow \widetilde{R},|-|_{R}$ : $R \longrightarrow R$ be maps such that $\left|r \cdot_{G} g\right|_{G}=|r|_{R} \cdot \widetilde{R}|g|_{G}$ for all $r \in R$ and all $g \in G$. Assume that any $\tau$-neighborhood of $0 \in R$ contains a ball $B_{\eta}^{R}(0):=\left\{\left.s \in R|| s\right|_{R}<_{R} \eta\right\}$ for some $\eta \in R, \eta>_{R} 0$, and that there exists some $\rho \in \widetilde{R}$ with $\rho>_{\widetilde{R}} 0$ such that the ball $B_{\rho}^{G}(0):=\left\{\left.g \in G|| g\right|_{G}<_{\widetilde{R}} \rho\right\}$ is $\sigma$-open. Finally, assume that

$$
\exists g \in G:|g|_{G} \text { is invertible, } \forall M \in \mathbb{R}_{>0}:|g|_{G}>_{\tilde{R}} \rho \cdot_{\tilde{R}} M .
$$

Then the induced topology $\tau \cap \mathbb{R}$ is discrete.

Proof. Since $G$ is a topological $R$-module, the product by scalars is $\tau \times \sigma$-continuous, and

$$
\lim _{\substack{r \rightarrow 0 \\ r \in R}} r \cdot{ }_{G} g=0,
$$

where $g \in G$ comes from assumption (5.5). By hypothesis, the ball $B_{\rho}^{G}(0) \in \sigma$ and every $\tau$-neighborhood of $r=0 \in R$ contains some ball $B_{\eta}^{R}(0)$. Therefore (5.6) entails that there exists some $\eta>_{R} 0$ such that

$$
\forall r \in R:|r|_{R}<_{R} \eta \quad \Longrightarrow \quad \rho>_{\widetilde{R}}\left|r \cdot_{G} g\right|_{G}=|r|_{R} \cdot \widetilde{R}|g|_{G}
$$

For each $s \in \mathbb{R}_{>0}$ take $M \in \mathbb{R}_{>0}$ such that $\frac{1}{M}<s$, so that $|g|_{G}>_{\widetilde{R}} \rho \cdot_{\tilde{R}} M>_{\widetilde{R}} 0$ from (5.5). For all $r \in R$ such that $|r|_{R}<_{R} \eta$, we have

$$
|r|_{R}<_{\widetilde{R}} \frac{\rho}{|g|_{G}}<_{\widetilde{R}} \frac{1}{M}<s
$$

because $|g|_{G}$ is invertible in $\widetilde{R}$. Therefore $|r|_{R}<_{\widetilde{R}} s$ and hence $|r|_{R}<_{R} s$ by (5.4). This means that $r$ is infinitesimal in the ring $R$, i.e. the ball $B_{\rho}^{R}(0)$ is contained in the monad of 0 (see e.g. [17] for the notion of monad) and so also every ball $B_{\eta}^{R}(\bar{r})$ is contained in the monad of $\bar{r} \in R$. Therefore, [17, Prop. 2.1] implies the conclusion.

We can therefore say that if we want to find a space $G$ of generalized functions which is an ordinary Hausdorff topological vector space on $\mathbb{R}$, then we cannot define the topologies $\tau$ and $\sigma$ using seminorms valued in a non-Archimedean (see (5.5)) extension of $\mathbb{R}$. This results confirms Rem. 43 of [10].

As a consequence, we have the following impossibility result: 
Corollary 44. There does not exist any real Hausdorff topological vector space $\left(G,+_{G},{ }_{G}, \sigma\right)$ such that:

(i) $\left(G,+_{G}, \cdot_{G}\right)$ is a linear subspace of $\mathcal{G D}_{K}(U)$ for some $\emptyset \neq K \Subset_{f} U \subseteq \widetilde{\mathbb{R}}^{n}$

(ii) $G$ contains the Dirac delta $\delta \in G$

(iii) $\exists m \in \mathbb{N} \exists \rho \in \widetilde{\mathbb{R}}_{>0}: \rho<1, B_{\rho}^{m}(0) \cap G \in \sigma$

In particular, the Colombeau algebra $\mathcal{G}^{s}(\Omega)$ does not contain any real Hausdorff topological vector subspace $G$ such that some $B_{\rho}^{m}(0) \cap G$ is open and $\delta \in G$.

Proof. By contradiction, in Thm. 43, set $R:=\mathbb{R}$ with the usual Euclidean topology $\tau, \widetilde{R}:=\widetilde{\mathbb{R}}$ with the sharp topology; set $|g|_{G}:=\|g\|_{m}$, where $m \in \mathbb{N}$ comes from (iii) and we used the inclusion (i); set $|r|_{R}:=|r|$ the usual absolute value in $\mathbb{R}$. Note also that $B_{\rho}^{m}(0) \cap G=\left\{g \in G \mid\|g\|_{m}<\rho\right\}=\left\{\left.g \in G|| g\right|_{G}<_{\tilde{R}} \rho\right\}$. If $\delta \in G$, then $|\delta|_{G}=\|\delta\|_{m}$ is infinite and invertible in $\widetilde{\mathbb{R}}$, so that Thm. 43 implies that the Euclidean topology would be discrete. The second part of the claim follows from Thm. 28.

We can summarize Cor. 44 by saying that a real Hausdorff topological vector space structure $G$ for a space of generalized functions cannot contain even a single trace $B_{\rho}^{m}(0) \cap G, \rho<1$, of a sharply open ball. This result does not contradict [1, Prop. 4], where it is stated that the sharp topology induces on bounded sets of the real locally convex space $\mathcal{G}_{a}(\Omega) \subseteq \mathcal{G}^{s}(\Omega)$ a topology which is finer than the topology $\sigma_{a}$ on $\mathcal{G}_{a}(\Omega)$. On the other hand, Cor. 44 implies that $B_{\rho}^{m}(0) \cap \mathcal{G}_{a}(\Omega) \notin \sigma_{a}$ for all $\rho \in \widetilde{\mathbb{R}}_{>0}, \rho<1$.

\section{Metric structure on $\mathcal{G} \mathcal{D}_{K}$}

In this section, we want to use [11, Thm. 1.14] to prove metrizability of $\mathcal{G D}_{K}(U)$. However, we will apply this result using an explicit and simple countable base of neighborhoods of the origin which consists of $\widetilde{\mathbb{R}}$-absorbent and absolutely $\widetilde{\mathbb{R}}$-convex sets. In this way, we will arrive at a simpler metric.

The idea is to consider only points of balls $f \in B_{\rho}^{m}(0)$ whose norm $\|f\|_{m}$ is infinitely smaller than $\rho$. To formally express this idea, we introduce the following

Definition 45. Let $\rho \in \widetilde{\mathbb{R}}_{>0}, m \in \mathbb{N}$ and $g \in \mathcal{G D}_{K}(U)$. Then

$$
U_{\rho}^{m}(g):=\left\{f \in \mathcal{G D}_{K}(U) \mid \frac{1}{\rho} \cdot\|f-g\|_{m} \approx 0\right\} .
$$

In case any confusion might arise, we will use the more precise symbol $U_{\rho}^{m}(g, K):=$ $U_{\rho}^{m}(g)$.

\section{Proposition 46.}

\footnotetext{
$U_{\rho}^{m}(0)$ is $\widetilde{\mathbb{R}}$-absorbent and absolutely $\widetilde{\mathbb{R}}$-convex.
} 
(ii) Both the system

$$
\left\{U_{\rho}^{m}(v) \mid v \in \mathcal{G} \mathcal{D}_{K}(U), m \in \mathbb{N}, \rho \in \widetilde{\mathbb{R}}_{>0}, \rho \approx 0\right\},
$$

and the system

$$
\left\{U_{\mathrm{d} \varepsilon^{n}}^{n}(v) \mid v \in \mathcal{G} \mathcal{D}_{K}(U), n \in \mathbb{N}_{>0}\right\}
$$

generate the sharp topology on $\mathcal{G D}_{K}(U)$.

Proof. To see that $U_{\rho}^{m}(0)$ is $\widetilde{\mathbb{R}}$-absorbent, by [11, Def. 1.1] we have to show that

$$
\forall u \in \mathcal{G D}_{K}(U) \exists a \in \mathbb{R} \forall b \in \mathbb{R}_{\leq a}: u \in \mathrm{d} \varepsilon^{b} \cdot U_{\rho}^{m}(0),
$$

i.e. $\frac{u}{\mathrm{~d} \varepsilon^{b}} \in U_{\rho}^{m}(0)$, which is equivalent to $\left\|\frac{u}{\mathrm{~d} \varepsilon^{b}}\right\|_{m} \cdot \frac{1}{\rho}=\frac{\|u\|_{m}}{\mathrm{~d} \varepsilon^{b} \cdot \rho} \approx 0$. But $\rho$ is strictly positive, so $\rho \geq \mathrm{d} \varepsilon^{p}$ for some $p \in \mathbb{R}$. Moreover, $\|u\|_{m} \in \widetilde{\mathbb{R}}$, so $\|u\|_{m} \leq \mathrm{d} \varepsilon^{q}$ for some $q \in \mathbb{R}$. Therefore

$$
\frac{\|u\|_{m}}{\mathrm{~d} \varepsilon^{b} \cdot \rho} \leq \mathrm{d} \varepsilon^{q-b-p}
$$

and we have $\mathrm{d} \varepsilon^{q-b-p} \approx 0$ if and only if $b<q-p$. This proves (6.1).

To prove that $U_{\rho}^{m}(0)$ is balanced, assume $\lambda \in \widetilde{\mathbb{R}}$ with $|\lambda|_{e} \leq 1$. Then $v(\lambda) \geq 0$, so $|\lambda| \leq c$ for some $c \in \mathbb{R}_{>0}$. Therefore, if $u \in U_{\rho}^{m}(0)$ then

$$
\frac{\|\lambda u\|_{m}}{\rho}=|\lambda| \frac{\|u\|_{m}}{\rho} \approx 0
$$

so $\lambda u \in U_{\rho}^{m}(0)$.

Finally, we show $\widetilde{\mathbb{R}}$-convexity: for all $a, b \in \mathbb{R}_{\geq 0}$ and all $u, v \in U_{\rho}^{m}(0)$, we have

$$
\frac{1}{\rho} \cdot\left\|\mathrm{d} \varepsilon^{a} \cdot u+\mathrm{d} \varepsilon^{b} \cdot v\right\|_{m} \leq \mathrm{d} \varepsilon^{a} \cdot \frac{\|u\|_{m}}{\rho}+\mathrm{d} \varepsilon^{b} \cdot \frac{\|v\|_{m}}{\rho} \approx 0 .
$$

In order to prove (ii), we note that $U_{\rho}^{m}(v) \subseteq B_{\rho}^{m}(v)$ because $\frac{\|u-v\|_{m}}{\rho} \approx 0$ implies $\frac{\|u-v\|_{m}}{\rho}<1$. Also, if $\rho \approx 0$, then $B_{\rho}^{m}(v) \subseteq U_{\sqrt{\rho}}^{m}(v)$ because $\|u-v\|_{m}<\rho$ implies $\frac{1}{\sqrt{\rho}} \cdot\|u-v\|_{m} \leq \sqrt{\rho} \approx 0$. Finally, if $\rho \approx 0$, every $U_{\rho}^{m}(v)$ is sharply open: if $u \in U_{\rho}^{m}(v)$ and $w \in B_{\rho^{2}}^{m}(u)$, then $\frac{1}{\rho} \cdot\|w-v\|_{m} \leq \frac{1}{\rho} \cdot\|w-u\|_{m}+\frac{1}{\rho} \cdot\|u-v\|_{m} \approx 0$. The proof for the second system in (ii) follows by observing that given $\rho>0, \rho \approx 0$, there exists $q \in \mathbb{N}$ such that $\rho \geq \mathrm{d} \varepsilon^{q}$, and setting $n:=\max (m, q)$ we have $U_{\mathrm{d} \varepsilon^{n}}^{n}(v) \subseteq U_{\rho}^{m}(v)$.

From [11, Thm. 1.14], we have that $\mathcal{G D}_{K}(U)$ is metrizable with metric

$$
d_{2}(u, v)=\sum_{n=1}^{+\infty} 2^{-n} \cdot \min \left\{\mathcal{P}_{U_{\mathrm{d} \varepsilon}^{n}(0)}(u-v), 1\right\} .
$$

Concerning (6.2) we recall (see [11]) that if $A \subseteq \mathcal{G D}_{K}(U)$ is $\widetilde{\mathbb{R}}$-absorbent, then, for all $u \in \mathcal{G D}_{K}(U)$, we define

$$
V_{A}(u):=\sup \left\{b \in \mathbb{R} \mid u \in \mathrm{d} \varepsilon^{b} \cdot A\right\}
$$




$$
\mathcal{P}_{A}(u):=e^{-V_{A}(u)} .
$$

The following result gives a metric which is equivalent to (6.2) but is defined by a simpler formula.

Proposition 47. Set $A_{n}:=U_{\mathrm{d} \varepsilon^{n}}^{n}(0)$ for $n \in \mathbb{N}_{>0}$, and let $u \in \mathcal{G} \mathcal{D}_{K}(U)$. Then

(i) $V_{A_{n}}(u)=v_{n}(u)-n$

(ii) The map

$$
d_{e}(u, v)=\sum_{n=1}^{+\infty} e^{\min \left[n-v_{n}(u-v), 0\right]-n} .
$$

is a metric on $\mathcal{G D}_{K}(U)$ that is equivalent to $d_{2}$.

Proof. Concerning (i), we note that

$$
\begin{aligned}
u \in \mathrm{d} \varepsilon^{b} \cdot A_{n} & \Rightarrow \frac{u}{\mathrm{~d} \varepsilon^{b}} \in U_{\mathrm{d} \varepsilon^{n}}^{n}(0) \Rightarrow \frac{\|u\|_{n}}{\mathrm{~d} \varepsilon^{b+n}} \approx 0 \Rightarrow v\left(\mathrm{~d} \varepsilon^{-b-n} \cdot\|u\|_{n}\right) \geq 0 \\
& \Rightarrow b \leq v_{n}(u)-n,
\end{aligned}
$$

so $V_{A_{n}}(u) \leq v_{n}(u)-n$. Conversely, if we had $V_{A_{n}}(u)<v_{n}(u)-n$, we could pick $V_{A_{n}}(u)<b<v_{n}(u)-n$. Then as above it would follow that $\frac{\|u\|_{n}}{\mathrm{~d} \varepsilon^{b+n}} \approx 0$, contradicting the definition of $V_{A_{n}}(u)$. This proves (i).

In order to prove (ii), we use (i) in (6.2): $\mathcal{P}_{A_{n}}(u-v)=e^{-v_{n}(u-v)+n}$ and

$$
\begin{aligned}
d_{2}(u, v) & =\sum_{n=1}^{+\infty} 2^{-n} \cdot \min \left\{e^{-v_{n}(u-v)+n}, 1\right\}= \\
& =\sum_{n=1}^{+\infty} 2^{-n} \cdot e^{\min \left[n-v_{n}(u-v), 0\right]} \geq \\
& \geq \sum_{n=1}^{+\infty} e^{\min \left[n-v_{n}(u-v), 0\right]-n}=d_{e}(u, v) .
\end{aligned}
$$

But we also have $e^{-n} \geq 2^{-n-1}$ for all $n$, so that $d_{e}(u, v) \geq \frac{1}{2} d_{2}(u, v)$. Using Prop. 40, it is easily checked that $d_{e}$ is a metric, which we have just proved to be equivalent to $d_{2}$.

\section{Completeness of $\mathcal{G} \mathcal{D}_{K}$}

In order to prove the completeness of $\mathcal{G D}_{K}(U)$, we generalize the proof of [11, Prop. 3.4] (based in turn on [28]) to the present context.

Theorem 48. The space $\mathcal{G D}_{K}(U)$ with the sharp topology is complete.

Proof. By Prop. $47 \mathcal{G D}_{K}(U)$ with the sharp topology is metrizable. Hence, it suffices to consider a Cauchy sequence $\left(u_{n}\right)_{n \in \mathbb{N}}$ in this topology, i.e.,

$$
\forall q \in \mathbb{R}_{>0} \forall i \in \mathbb{N} \exists N \in \mathbb{N} \forall m, n \geq N:\left\|u_{n}-u_{m}\right\|_{i}<\mathrm{d} \varepsilon^{q} .
$$


Setting $i=q=k \in \mathbb{N}_{>0}$, this implies the existence of a strictly increasing sequence $\left(n_{k}\right)_{k \in \mathbb{N}}$ in $\mathbb{N}$ such that $\left\|u_{n_{k+1}}-u_{n_{k}}\right\|_{k}<\mathrm{d} \varepsilon^{k}$. Hence picking any representatives $\left(u_{n \varepsilon}\right)$ of $u_{n}$ as in Def. 19 we have

$$
\left[\max _{|\alpha| \leq k} \sup _{x \in \mathbb{R}^{n}}\left|\partial^{\alpha} u_{n_{k+1}, \varepsilon}(x)-\partial^{\alpha} u_{n_{k}, \varepsilon}(x)\right|\right]<\left[\varepsilon^{k}\right] \quad \forall k \in \mathbb{N}_{>0} .
$$

By Lemma 1 this yields that for each $k \in \mathbb{N}_{>0}$ there exists an $\varepsilon_{k}$ such that $\varepsilon_{k} \searrow 0$ and

$$
\forall \varepsilon \in\left(0, \varepsilon_{k}\right): \max _{|\alpha| \leq k} \sup _{x \in \mathbb{R}^{n}}\left|\partial^{\alpha} u_{n_{k+1}, \varepsilon}(x)-\partial^{\alpha} u_{n_{k}, \varepsilon}(x)\right|<\varepsilon^{k}
$$

Now set

$$
\begin{gathered}
h_{k \varepsilon}:= \begin{cases}u_{n_{k+1}, \varepsilon}-u_{n_{k}, \varepsilon} \in \mathcal{C}^{\infty}\left(\mathbb{R}^{n}, \mathbb{R}\right) & \text { if } \varepsilon \in\left(0, \varepsilon_{k}\right) \\
0 \in \mathcal{C}^{\infty}\left(\mathbb{R}^{n}, \mathbb{R}\right) & \text { if } \varepsilon \in\left[\varepsilon_{k}, 1\right)\end{cases} \\
u_{\varepsilon}:=u_{n_{0} \varepsilon}+\sum_{k=0}^{\infty} h_{k \varepsilon} \quad \forall \varepsilon \in I .
\end{gathered}
$$

Since $\varepsilon_{k} \searrow 0$, for all $\varepsilon \in I$ we have $\varepsilon \notin\left(0, \varepsilon_{k}\right)$ for all $k \geq \bar{k}$, with $\bar{k}$ sufficiently big. Therefore, $u_{\varepsilon}=u_{n_{\bar{k}+1}, \varepsilon} \in \mathcal{C}^{\infty}\left(\mathbb{R}^{n}, \mathbb{R}\right)$. In order to prove that $\left(u_{\varepsilon}\right)$ defines a GSF of the type $U \rightarrow \widetilde{\mathbb{R}}$, take $\left[x_{\varepsilon}\right] \in U$ and $\alpha \in \mathbb{N}$. We claim that $\left(\partial^{\alpha} u_{\varepsilon}\left(x_{\varepsilon}\right)\right) \in \mathbb{R}_{M}$. Now if $p \in \mathbb{N}$ satisfies $|\alpha| \leq p$, then for any $x \in \mathbb{R}^{n}$ we have

$$
\left|\partial^{\alpha} u_{\varepsilon}(x)\right| \leq\left|\partial^{\alpha} u_{n_{p+1}, \varepsilon}(x)\right|+\sum_{k=p+1}^{\infty}\left|\partial^{\alpha} h_{k \varepsilon}(x)\right| .
$$

From (7.1) and (7.2) we get that $\left|\partial^{\alpha} h_{k \varepsilon}(x)\right| \leq \varepsilon^{k}$ for all $k \geq p+1, x \in \mathbb{R}^{n}$ and all $\varepsilon \in(0,1]$. Hence for $\varepsilon \in(0,1],|\alpha| \leq p$ and all $x \in \mathbb{R}^{n}$ we obtain

$$
\left|\partial^{\alpha} u_{\varepsilon}(x)\right| \leq\left|\partial^{\alpha} u_{n_{p+1}, \varepsilon}(x)\right|+\frac{\varepsilon^{p+1}}{1-\varepsilon}
$$

Inserting $x=x_{\varepsilon}$ and noting that $\left(\partial^{\alpha} u_{n_{p+1}, \varepsilon}\left(x_{\varepsilon}\right)\right) \in \mathbb{R}_{M}$ proves our claim. Moreover, since all $\left(u_{n \varepsilon}\right)$ satisfy Def. 19, we also conclude from (7.3) that for any $\alpha$ and any $\left[x_{\varepsilon}\right] \in \operatorname{ext}(K)$ we have $\left[\partial^{\alpha} u_{\varepsilon}\left(x_{\varepsilon}\right)\right]=0$, and hence the $\left.\operatorname{GSF}\left[u_{\varepsilon}(-)\right]\right|_{U} \in \mathcal{G} \mathcal{D}_{K}(U)$.

Finally, $\left\|u-u_{n_{p}}\right\|_{i}<\mathrm{d} \varepsilon^{p-1}$ for all $p \in \mathbb{N}_{>1}$ and all $i \leq p$. This yields that $\left(u_{n_{k}}\right)_{k}$ tends to $u$ in the sharp topology, and hence so does $\left(u_{n}\right)$.

\section{The space $\mathcal{G} \mathcal{D}$ as inductive limit of $\mathcal{G D}_{K}$}

In this section, we always assume that $U \subseteq \widetilde{\mathbb{R}}^{n}$ is a non-empty strongly internal set. By Prop. 35 and Prop. 36, this entails that $\mathcal{G D}(U)$ is an $\widetilde{\mathbb{R}}$-module.

In order to define a natural topology on $\mathcal{G D}(U)$ we will employ [11, Thm. 1.18], which we restate here for the reader's convenience:

Theorem 49. Let $\mathcal{G}$ be an $\widetilde{\mathbb{R}}$-module, $\left(\mathcal{G}_{\gamma}\right)_{\gamma \in \Gamma}$ be a family of locally convex topological $\widetilde{\mathbb{R}}$-modules and, for each $\gamma \in \Gamma$, let $i_{\gamma}: \mathcal{G}_{\gamma} \longrightarrow \mathcal{G}$ be an $\widetilde{\mathbb{R}}$-linear map. Assume 
that

$$
\mathcal{G}=\operatorname{span}\left(\bigcup_{\gamma \in \Gamma} i_{\gamma}\left(\mathcal{G}_{\gamma}\right)\right)
$$

and let $V \in \mathcal{V}$ if and only if $V \subseteq \mathcal{G}, V$ is absolutely $\widetilde{\mathbb{R}}$-convex and $i_{\gamma}^{-1}(V)$ is a neighborhood of 0 in $\mathcal{G}_{\gamma}$ for all $\gamma \in \Gamma$. Then each $V \in \mathcal{V}$ is $\widetilde{\mathbb{R}}$-absorbent and the topology $\tau$ induced by the gauges $\left\{\mathcal{P}_{V}\right\}_{V \in \mathcal{V}}$ (see [11] and Def. (6.3)) is the finest $\widetilde{\mathbb{R}}$-locally convex topology on $\mathcal{G}$ such that $i_{\gamma}$ is continuous for all $\gamma \in \Gamma$. Endowed with this topology, $\mathcal{G}$ is called the inductive limit (colimit) of the spaces $\left(\mathcal{G}_{\gamma}\right)_{\gamma \in \Gamma}$ and we write $\mathcal{G}=\underset{\lim }{\longrightarrow} \mathcal{G}_{\gamma}$.

Since $\mathcal{G} \mathcal{D}(U)=\bigcup_{\emptyset \neq K \Subset_{\mathrm{f}} U} \mathcal{G} \mathcal{D}_{K}(U)$, we may therefore equip it with the inductive limit topology with respect to the inclusions $\iota_{K}: \mathcal{G D}_{K}(U) \hookrightarrow \mathcal{G D}(U)$. We call the resulting $\widetilde{\mathbb{R}}$-locally convex topology the sharp topology on $\mathcal{G} \mathcal{D}(U)$. Hence

$$
\mathcal{G D}(U)=\lim _{\longrightarrow} \mathcal{G} \mathcal{D}_{K}(U) \quad\left(\emptyset \neq K \Subset_{\mathrm{f}} U\right) .
$$

Henceforth we will denote the sharp topology on $\mathcal{G D}_{K}(U)$ by $\sigma_{K}(U)$ (or, for short, by $\sigma_{K}$ ). Also, the inductive limit topology on $\mathcal{G D}(U)$ will be denoted by $\sigma(U)$ (or by $\sigma)$. Setting

$$
U_{\mathrm{d} \varepsilon^{n}}^{n}(0):=\left\{f \in \mathcal{G} \mathcal{D}(U) \mid \frac{\|f\|_{n}}{\mathrm{~d} \varepsilon^{n}} \approx 0\right\},
$$

where $n \in \mathbb{N}_{>0}$, we obtain an $\widetilde{\mathbb{R}}$-absorbent and absolutely $\widetilde{\mathbb{R}}$-convex subset of $\mathcal{G D}(U)$ such that $i_{K}^{-1}\left(U_{\mathrm{d} \varepsilon^{n}}^{n}(0)\right)=U_{\mathrm{d} \varepsilon^{n}}^{n}(0) \cap \mathcal{G D}_{K}(U)=U_{\mathrm{d} \varepsilon^{n}}^{n}(0, K) \in \sigma_{K}$. Therefore, these sets generate a coarser topology than the sharp topology $\sigma$. The proof is identical to that of Prop. 46 (i).

From the (co-) universal property of inductive limits ([11, Prop. 1.19]) we immediately conclude:

Proposition 50. Let $\mathcal{H}$ be a locally convex topological $\widetilde{\mathbb{R}}-$ module. For each nonempty $K \Subset_{f} U$, let $T_{K}: \mathcal{G D}_{K}(U) \longrightarrow \mathcal{H}$ be an $\widetilde{\mathbb{R}}$-linear and continuous map. Assume that $T_{K}(f)=T_{H}(f)$ if $f \in \mathcal{G D}_{K}(U) \cap \mathcal{G D}_{H}(U)$. Then there exists one and only one map $T: \mathcal{G D}(U) \longrightarrow \mathcal{H}$ which is $\widetilde{\mathbb{R}}$-linear and continuous and such that $T \circ \iota_{K}=T_{K}$ for all non-empty $K \Subset_{f} U$.

The work [11] includes a detailed analysis of countable inductive limits $\mathcal{G}=$ $\lim _{\longrightarrow} \mathcal{G}_{n}(n \in \mathbb{N})$, where $\left(\mathcal{G}_{n}\right)_{n \in \mathbb{N}}$ is increasing, $\mathcal{G}=\bigcup_{n \in \mathbb{N}} \mathcal{G}_{n}$, and where the topology $\overrightarrow{\text { on }} \mathcal{G}_{n}$ is that induced by $\mathcal{G}_{n+1}$. Such inductive limits are called strict. As in the case of classical function spaces like $\mathcal{D}(\Omega)$ for $\Omega$ open in $\mathbb{R}^{n}$, the importance of strict inductive limits in the theory of $\widetilde{\mathbb{R}}$-locally convex models ultimately stems from the possibility of covering every open set $\Omega \subseteq \mathbb{R}^{n}$ by a countable increasing family of compact sets. The key point in the structure theory of strict inductive limits as above is that a countable family $\left(\mathcal{G}_{n}\right)_{n \in \mathbb{N}}$ permits to define recursively a 
family of neighborhoods of 0 . Using the latter, one can prove that the topology on $\mathcal{G}$ induces on each $\mathcal{G}_{n}$ its given topology. We will show below that similar properties hold for $\mathcal{G D}(U)$. We shall see that the assumption of $U$ being strongly internal and sharply open are essential for this task. To begin with, we prove a strengthening of Thm. 29:

Proposition 51. Let $H, K \Subset_{f} U$ be non-empty sets, with $H \subseteq K$. Then $\mathcal{G D}_{H}(U)$ is a topological subspace of $\mathcal{G} \mathcal{D}_{K}(U)$, i.e.,

$$
\left.\sigma_{K}(U)\right|_{\mathcal{G D}_{H}(U)}=\sigma_{H}(U) .
$$

Proof. In this proof we will use the more precise notation $B_{\rho}^{m}(u, K)$ for balls (see Def. 38).

Let $V \in \sigma_{K}$. We claim that $V \cap \mathcal{G D}_{H}(U) \in \sigma_{H}$. For each $u \in V \cap \mathcal{G D}_{H}(U)$ there exist $m \in \mathbb{N}$ and $\rho \in \widetilde{\mathbb{R}}_{>0}$ such that $B_{\rho}^{m}(u, K) \subseteq V$. But $B_{\rho}^{m}(u, H) \subseteq B_{\rho}^{m}(u, K)$ because $\mathcal{G D}_{H}(U) \subseteq \mathcal{G D}_{K}(U)$ and because the norm $\|-\|_{m}$ doesn't depend on $H$, $K$. Therefore, $B_{\rho}^{m}(u, H) \subseteq V \cap \mathcal{G D}_{H}(U)$, which proves our claim.

Conversely, if $W \in \sigma_{H}$, then we set

$$
V:=\bigcup\left\{B_{\rho}^{m}(u, K) \mid u \in W, m \in \mathbb{N}, \rho \in \widetilde{\mathbb{R}}_{>0}, B_{\rho}^{m}(u, H) \subseteq W\right\} \in \sigma_{K},
$$

and we claim that $W=V \cap \mathcal{G D}_{H}(U)$. In fact, since $W \in \sigma_{H}$, for all $u \in W$ we have $B_{\rho}^{m}(u, H) \subseteq W$ for some $\rho$ and $m$. By (8.1) $B_{\rho}^{m}(u, K) \subseteq V$, and so $u \in V \cap \mathcal{G} \mathcal{D}_{H}(U)$. Vice versa, if $u \in V \cap \mathcal{G D}_{H}(U)$, then $u \in B_{\rho}^{m}(v, K)$ for some $v \in W, m, \rho$, such that $B_{\rho}^{m}(v, H) \subseteq W$. So $\|u-v\|_{m}<\rho$ and hence $u \in B_{\rho}^{m}(v, H) \subseteq W$.

We now show that the space $\mathcal{G} \mathcal{D}(U)$ can be seen as a strict inductive limit of a countable increasing family of subspaces $\mathcal{G D}_{K}(U)$. Indeed, since $U$ is strongly internal, we can write $U=\left\langle U_{\varepsilon}\right\rangle$ for some net $\left(U_{\varepsilon}\right)$ of subsets of $\mathbb{R}^{n}$. Since $U$ is non-empty, by [18, Thm. 8], fixing any $x=\left[x_{\varepsilon}\right] \in U$ we obtain:

$$
\exists N \in \mathbb{N} \forall^{0} \varepsilon: d\left(x_{\varepsilon}, U_{\varepsilon}^{c}\right)>\varepsilon^{N},\left|x_{\varepsilon}\right| \leq \varepsilon^{-N} .
$$

With $N$ as in (8.2), we define

$$
\begin{aligned}
K_{j \varepsilon} & :=\left\{x \in \mathbb{R}^{n}\left|d\left(x, U_{\varepsilon}^{c}\right) \geq \varepsilon^{j},\right| x \mid \leq \varepsilon^{-j}\right\} \\
K_{j} & :=\left[K_{j \varepsilon}\right] \quad \forall j \in \mathbb{N}_{\geq N} .
\end{aligned}
$$

Using this notation, we have:

Theorem 52. If $N \in \mathbb{N}$ satisfies (8.2) for some $x \in U$, then

$$
\emptyset \neq K_{j} \Subset_{f} U \text { for all } j \in \mathbb{N}_{\geq N}
$$

(ii) $K_{j} \subseteq K_{j+1}$ for all $j \in \mathbb{N}_{\geq N}$

(iii) $U=\bigcup_{j \geq N} K_{j}$

(iv) For every $\emptyset \neq K \Subset_{f} U=\left\langle U_{\varepsilon}\right\rangle$ there exists some $j \geq N$ such that $K \subseteq K_{j}$. 
(v) $\mathcal{G D}(U)$ is the strict inductive limit of the family $\mathcal{G D}_{K_{j}}(U), j \geq N$ :

$$
\mathcal{G D}(U)=\lim _{\longrightarrow} \mathcal{G D}_{K_{j}}(U) \quad(j \geq N) .
$$

Proof. (i), (ii): It follows immediately from the definition that each $\left(K_{j \varepsilon}\right)$ is compact and that $\left(K_{j \varepsilon}\right)_{\varepsilon}$ is sharply bounded, so $K_{j} \Subset_{\mathrm{f}} U$. Moreover, $K_{N} \neq \emptyset$ by (8.2), hence $K_{j} \neq \emptyset$ follows by (ii), which again is immediate from the definition.

(iii): If $x=\left[x_{\varepsilon}\right] \in U=\left\langle U_{\varepsilon}\right\rangle$, then $d\left(x_{\varepsilon}, U_{\varepsilon}^{c}\right)>\varepsilon^{j_{1}}$ for $\varepsilon$ small and for some $j_{1} \in \mathbb{N}_{>0}$. Since $\left(x_{\varepsilon}\right)$ is moderate, $\left|x_{\varepsilon}\right| \leq \varepsilon^{-j_{2}}$ for $\varepsilon$ small and some $j_{2} \in \mathbb{N}$. Setting $j:=\max \left(j_{1}, j_{2}, N\right)$ we hence have that $x \in K_{j}$.

In order to prove (iv), we need the following strengthening of [18, Thm. 11]:

Lemma 53. Let $H=\left[H_{\varepsilon}\right] \Subset_{f} V=\left\langle V_{\varepsilon}\right\rangle$, then

$$
\exists j \in \mathbb{N} \forall\left[x_{\varepsilon}\right] \in\left[H_{\varepsilon}\right] \forall^{0} \varepsilon: d\left(x_{\varepsilon}, V_{\varepsilon}^{c}\right) \geq \varepsilon^{j} .
$$

Proof of Lemma 53. Equation (8.3) expresses that for all representatives $\left(x_{\varepsilon}\right) \in$ $\mathbb{R}_{M}^{n}$, if $x_{\varepsilon} \in H_{\varepsilon}$ for $\varepsilon$ small, then $\forall^{0} \varepsilon: d\left(x_{\varepsilon}, V_{\varepsilon}^{c}\right) \geq \varepsilon^{j}$.

By contradiction, assume that

$$
\forall j \in \mathbb{N} \exists\left(x_{j \varepsilon}\right) \in \mathbb{R}_{M}^{n}:\left(\forall^{0} \varepsilon: x_{j \varepsilon} \in H_{\varepsilon}\right), \exists\left(\varepsilon_{j k}\right)_{k} \downarrow 0 \forall k: d\left(x_{j \varepsilon_{j k}}, V_{\varepsilon_{j k}}^{c}\right)<\varepsilon_{j k}^{j} .
$$

By recursively applying this condition, we get that for all $j \in \mathbb{N}$ there exists a moderate $\left(x_{j \varepsilon}\right)$ and some $\varepsilon_{j} \in(0,1]$ such that $x_{j \varepsilon} \in H_{\varepsilon}$ for $\varepsilon \leq \varepsilon_{j}$ and

$$
d\left(x_{j \varepsilon_{j k}}, V_{\varepsilon_{j k}}^{c}\right)<\varepsilon_{j k}^{j},
$$

where $\left(\varepsilon_{j k}\right)_{k} \downarrow 0$. Since $\left(\varepsilon_{j k}\right)_{k} \downarrow 0$, without loss of generality we can assume to have defined recursively $\left(\varepsilon_{j}\right)_{j}$ so that $\left(\varepsilon_{j}\right)_{j} \downarrow 0$ and $\varepsilon_{j}>\varepsilon_{j k_{j}}>\varepsilon_{j+1}$ for some subsequence $\left(k_{j}\right)_{j} \uparrow+\infty$. Set $x_{\varepsilon}:=x_{j \varepsilon} \in H_{\varepsilon}$ for $\varepsilon \in\left(\varepsilon_{j+1}, \varepsilon_{j}\right]$, so that $x_{\varepsilon_{j k_{j}}}=x_{j \varepsilon_{j k_{j}}}$ for all $j$. Then $\left(x_{\varepsilon}\right) \in \mathbb{R}_{M}^{n}$ since $H$ is sharply bounded and $x:=\left[x_{\varepsilon}\right] \in H \subseteq\left\langle V_{\varepsilon}\right\rangle$, which entails

$$
\exists q \in \mathbb{R}_{>0} \forall^{0} \varepsilon: d\left(x_{\varepsilon}, V_{\varepsilon}^{c}\right)>\varepsilon^{q} .
$$

Therefore, for $j \in \mathbb{N}$ sufficiently big, (8.5) holds at $\varepsilon=\varepsilon_{j k_{j}}<1$ and $j>q$. Thus $d\left(x_{j \varepsilon_{j k_{j}}}, V_{\varepsilon_{j k_{j}}}^{c}\right)=d\left(x_{\varepsilon_{j k_{j}}}, V_{\varepsilon_{j k_{j}}}^{c}\right)>\varepsilon_{j k_{j}}^{q}>\varepsilon_{j k_{j}}^{j}$, which contradicts (8.4).

Continuing the proof of (iv), if $K \Subset_{\mathrm{f}} U$ is non-empty, by applying Lemma 53 we obtain

$$
\exists j_{1} \in \mathbb{N} \forall\left[x_{\varepsilon}\right] \in K \forall^{0} \varepsilon: d\left(x_{\varepsilon}, U_{\varepsilon}^{c}\right) \geq \varepsilon^{j_{1}} .
$$

On the other hand, sharp boundedness of $\left(K_{\varepsilon}\right)$, where $K=\left[K_{\varepsilon}\right]$, implies

$$
\exists j_{2} \in \mathbb{N} \forall\left[x_{\varepsilon}\right] \in K \forall^{0} \varepsilon:\left|x_{\varepsilon}\right| \leq \varepsilon^{-j_{2}} .
$$


Therefore, for $j:=\max \left(j_{1}, j_{2}, N\right)$, we get $K \subseteq K_{j}$, hence (iv), and thereby also $\mathcal{G D}_{K}(U) \subseteq \mathcal{G D}_{K_{j}}(U)$ (using Thm. 29). It follows that $\mathcal{G D}(U) \subseteq \bigcup_{j \geq N} \mathcal{G D}_{K_{j}}(U)$. The converse inclusion follows directly from (i).

It remains to prove that the topology $\sigma$ on $\mathcal{G D}(U)$ coincides with the inductive $\widetilde{\mathbb{R}}$-locally convex topology generated by $\left(\mathcal{G} \mathcal{D}_{K_{j}}(U)\right)_{j \geq N}$. Let us denote the latter topology by $\sigma^{\prime}$. We have $\sigma \subseteq \sigma^{\prime}$ by definition of the inductive topology and by Thm. 52.(i). To see that, conversely, $\sigma^{\prime} \subseteq \sigma$ we show that for every $K \Subset_{\mathrm{f}} U$ the inclusion $\left(\mathcal{G D}_{K}(U), \sigma_{K}\right) \hookrightarrow\left(\mathcal{G D}(U), \sigma^{\prime}\right)$ is continuous (see Thm. 49). Now given any $K \Subset_{\mathrm{f}} U$, by what we have proved above there exists some $j \geq N$ with $K \subseteq K_{j}$. But then Prop. 51 implies the continuity of

$$
\left(\mathcal{G D}_{K}(U), \sigma_{K}\right) \hookrightarrow\left(\mathcal{G D}_{K_{j}}(U), \sigma_{K_{j}}\right) \hookrightarrow\left(\mathcal{G D}(U), \sigma^{\prime}\right)
$$

and thereby our claim.

As a consequence of this result, we can now prove a series of corollaries by applying the general theorems of [11] concerning countable strict inductive limits.

Corollary 54. If $\emptyset \neq K \Subset_{f} U=\left\langle U_{\varepsilon}\right\rangle$, then $\mathcal{G D}_{K}(U)$ is a topological subspace of $\mathcal{G D}(U)$, i.e., $\left.\sigma(U)\right|_{\mathcal{G D}_{K}(U)}=\sigma_{K}(U)$.

Proof. According to Thm. 52 (iv) we may pick $j \geq N$ such that $K \subseteq K_{j}$. By [11, Prop. 1.21], $\mathcal{G D}_{K_{j}}(U)$ carries the trace topology of $\mathcal{G D}(U)$. Since, in turn, $\mathcal{G D}_{K}(U)$ is a topological subspace of $\mathcal{G} \mathcal{D}_{K_{j}}(U)$ by Prop. 51, the claim follows.

Corollary 55. $\mathcal{G D}(U)$ is separated.

Proof. This follows from Thm. 41 (v) and [11, Cor. 1.24].

Lemma 56. If $\emptyset \neq H, K \Subset_{f} U$ and $H \subseteq K$, then $\mathcal{G D}_{H}(U)$ is closed in $\mathcal{G D}_{K}(U)$.

Proof. This is immediate from Prop. 51 and Thm. 48.

Corollary 57. If $\emptyset \neq K \Subset_{f} U$, then $\mathcal{G D}_{K}(U)$ is closed in $\mathcal{G D}(U)$.

Proof. This follows from Thm. 48, Cor. 55, and Cor. 54.

Corollary 58. Let $B \subseteq \mathcal{G D}(U)$, then $B$ is bounded in $\mathcal{G D}(U)$ if and only if there exists a non-empty $K \Subset_{f} U$ such that $B$ is bounded in $\mathcal{G D}_{K}(U)$.

Proof. $\Rightarrow$ : This follows from [11, Thm. 1.26] and Lemma 56.

$\Leftarrow$ : If $B$ is bounded in $\mathcal{G D}_{K}(U)$, then [11, Lem. 1.27] yields

$$
\forall\left(u_{n}\right)_{n} \in B^{\mathbb{N}} \forall\left(\lambda_{n}\right)_{n} \in \widetilde{\mathbb{R}}^{\mathbb{N}}: \lambda_{n} \rightarrow 0 \text { in } \widetilde{\mathbb{R}} \Longrightarrow \lambda_{n} u_{n} \rightarrow 0 \text { in } \mathcal{G D}_{K}(U) .
$$

Pick $j \geq N$ such that $K \subseteq K_{j}$. Since generalized norms $\|-\|_{m}$ do not depend on $K, K_{j}$, condition (8.6) holds also in $\mathcal{G D}_{K_{j}}(U) \supseteq \mathcal{G D}_{K}(U)$. Therefore, from [11, 
Lem. 1.27] we get that $B$ is bounded in $\mathcal{G D}_{K_{j}}(U)$ and [11, Thm. 1.26] yields that $B$ is bounded in $\mathcal{G} \mathcal{D}(U)$.

A similar proof applies to this corollary, which is a consequence of [11, Cor. 1.29]:

Corollary 59. Let $\left(u_{n}\right)_{n} \in \mathcal{G D}(U)^{\mathbb{N}}$, then $u_{n} \rightarrow 0$ in $\mathcal{G D}(U)$ if and only if there exists a non-empty $K \Subset_{f} U$ such that $u_{n} \in \mathcal{G D}_{K}(U)$ and $u_{n} \rightarrow 0$ in $\mathcal{G D}_{K}(U)$.

Finally, from [11, Thm. 1.32], Lemma 56 and Thm. 48 we obtain:

Corollary 60. $\mathcal{G D}(U)$ with the sharp topology is complete.

Using Lemma 53, we can also show that any compactly supported generalized smooth function $f \in \mathcal{G D}_{K}(U, Y)$ on a sharply open set $U \subseteq \widetilde{\mathbb{R}}^{n}$ is defined by a net $\left(u_{\varepsilon}\right)$ of smooth functions which are compactly supported in an arbitrarily small extension $\left[K_{\varepsilon}+\overline{B_{\varepsilon^{a}}^{\mathrm{E}}(0)}\right]$ of $K=\left[K_{\varepsilon}\right]$. We recall that in this section we are assuming that $U$ is a strongly internal set.

Theorem 61. Let $\emptyset \neq K \Subset_{f} U$. Let $Y \subseteq \widetilde{\mathbb{R}}^{d}, f \in \mathcal{G} \mathcal{D}_{K}(U, Y)$ and $K=\left[K_{\varepsilon}\right] \Subset_{f} \widetilde{\mathbb{R}}^{n}$. Let $j \in \mathbb{N}$ be as in (8.3) and $a \in \mathbb{R}$ such that $a \geq j$. Then there exist nets $\left(u_{\varepsilon}\right)$, $\left(H_{\varepsilon}\right)$ such that:

(i) $\left[H_{\varepsilon}\right] \Subset_{f} U$

(ii) $\left(u_{\varepsilon}\right)$ defines $f$ and $u_{\varepsilon} \in \mathcal{D}_{H_{\varepsilon}}\left(\mathbb{R}^{n}, \mathbb{R}^{d}\right)$ for all $\varepsilon$

(iii) $H_{\varepsilon} \subseteq K_{\varepsilon}+\overline{B_{\varepsilon^{a}}^{\mathrm{E}}(0)}$ for all $\varepsilon$.

Proof. Let $U=\left\langle U_{\varepsilon}\right\rangle$, where each $U_{\varepsilon} \subseteq \mathbb{R}^{n}$ is an open set (cf. [18, Cor. 9]), and let $\left(v_{\varepsilon}\right)$ satisfy Def. 19 for $f$ and $K=\left[K_{\varepsilon}\right]$. By [18, Thm. 11], we can assume $K_{\varepsilon} \subseteq U_{\varepsilon}$ for all $\varepsilon$. Let $L_{\varepsilon}:=K_{\varepsilon}+\overline{B_{\varepsilon^{a} / 2}^{\mathrm{E}}(0)}$, and denote by $\chi_{L_{\varepsilon}}$ the characteristic function of $L_{\varepsilon}$. Let $\psi \in \mathcal{D}\left(B_{1}^{\mathrm{E}}(0)\right)$ have unit integral and set $\psi_{\varepsilon}:=\left(\varepsilon^{a} / 2\right)^{-n} \psi\left(2 x / \varepsilon^{a}\right)$. Then $\left(\psi_{\varepsilon}\right) \in \mathcal{E}_{M}^{s}\left(\mathbb{R}^{n}\right)$, and $\left.\left(\chi_{L_{\varepsilon}} * \psi_{\varepsilon^{a}}\right)\right|_{K_{\varepsilon}}=1$. Set $u_{\varepsilon}:=\left(\chi_{L_{\varepsilon}} * \psi_{\varepsilon}\right) \cdot v_{\varepsilon}$. Then $\left(\chi_{L_{\varepsilon}} * \psi_{\varepsilon}\right)$ defines a GSF of the type $\widetilde{\mathbb{R}}^{n} \longrightarrow \widetilde{\mathbb{R}}$ and hence $\left(u_{\varepsilon}\right)$ defines a GSF of the type $U \longrightarrow \widetilde{\mathbb{R}}^{d}$. Moreover, $H_{\varepsilon}:=\operatorname{supp}\left(u_{\varepsilon}\right) \subseteq \operatorname{supp}\left(\chi_{L_{\varepsilon}} * \psi_{\varepsilon}\right) \subseteq K_{\varepsilon}+\overline{B_{\varepsilon^{a}}^{\mathrm{E}}(0)}$. Since $a \geq j$, we have $\left[H_{\varepsilon}\right] \subseteq\left\langle U_{\varepsilon}\right\rangle=U$.

It remains to prove that $f(x)=\left[u_{\varepsilon}\left(x_{\varepsilon}\right)\right]$ for all $x=\left[x_{\varepsilon}\right] \in U$. Suppose this was not the case. Then there would exist some $y=\left[y_{\varepsilon}\right] \in U$, some $b>0$ and a sequence $\varepsilon_{k} \searrow 0$ such that

$$
\left|u_{\varepsilon_{k}}\left(y_{\varepsilon_{k}}\right)-v_{\varepsilon_{k}}\left(y_{\varepsilon_{k}}\right)\right| \geq \varepsilon_{k}^{b}
$$

for all $k \in \mathbb{N}$. By Lemma 21, we may without loss of generality assume that either $y_{\varepsilon} \in K_{\varepsilon}$ for all $\varepsilon$ or that $y \in \operatorname{ext}(K)$. In the first case, $v_{\varepsilon}\left(y_{\varepsilon}\right)=u_{\varepsilon}\left(y_{\varepsilon}\right)$ for all $\varepsilon$, contradicting (8.7). In the second case,

$$
\left|v_{\varepsilon}\left(y_{\varepsilon}\right)-u_{\varepsilon}\left(y_{\varepsilon}\right)\right| \leq 2\left|v_{\varepsilon}\left(y_{\varepsilon}\right)\right| .
$$

Since $\left.\left(v_{\varepsilon}\left(y_{\varepsilon}\right)\right)\right)$ is negligible, we again arrive at a contradiction to (8.7). 
Assume that we have an operator $I: \mathcal{D}\left(\mathbb{R}^{n}\right) \longrightarrow \mathbb{R}$ with the property that if $\left(u_{\varepsilon}\right)$ and $\left(v_{\varepsilon}\right)$ define $f \in \mathcal{G D}(U)$, where $u_{\varepsilon}, v_{\varepsilon} \in \mathcal{D}\left(\mathbb{R}^{n}\right)$, then $\left[I\left(u_{\varepsilon}\right)\right]=\left[I\left(v_{\varepsilon}\right)\right] \in \widetilde{\mathbb{R}}$. Then Thm. 61 permits to extend $I$ to the whole of $\mathcal{G D}(U)$.

Using this result, we can now prove the extension of property (ii) of Thm. 26 to arbitrary codomains $Y \subseteq \widetilde{\mathbb{R}}^{d}$ :

Theorem 62. Let $\emptyset \neq K \Subset_{f} U, Y \subseteq \widetilde{\mathbb{R}}^{d}$ and $f \in \mathcal{G D}_{K}(U, Y)$, then $\exists ! \bar{f} \in$ $\mathcal{G D}^{\mathrm{g}}(K, Y):\left.\bar{f}\right|_{K}=\left.f\right|_{K}$.

Proof. We only have to prove that $\bar{f}(x) \in Y$ for all $x \in \widetilde{\mathbb{R}}^{n}$. Let $\left(u_{\varepsilon}\right)$ and $\left(H_{\varepsilon}\right)$ as in Thm. 61. By Thm. 24, we have that $\left(u_{\varepsilon}\right)$ also defines $\bar{f}$. For each $\varepsilon$ pick any point $h_{\varepsilon} \in \partial H_{\varepsilon}$ and set $\bar{x}_{\varepsilon}:=x_{\varepsilon}$ if $x_{\varepsilon} \in H_{\varepsilon}$, and $\bar{x}_{\varepsilon}:=h_{\varepsilon}$ otherwise. Therefore $\bar{x}:=\left[\bar{x}_{\varepsilon}\right] \in\left[H_{\varepsilon}\right] \subseteq U$. Then, if $x_{\varepsilon} \notin H_{\varepsilon},\left|u_{\varepsilon}\left(x_{\varepsilon}\right)-u_{\varepsilon}\left(\bar{x}_{\varepsilon}\right)\right|=\left|u_{\varepsilon}\left(x_{\varepsilon}\right)-u_{\varepsilon}\left(h_{\varepsilon}\right)\right|=0$ because $u_{\varepsilon} \in \mathcal{D}_{H_{\varepsilon}}\left(\mathbb{R}^{n}, \mathbb{R}^{d}\right)$. Thus $\bar{f}(x)=f(\bar{x}) \in Y$.

\section{Conclusions and Further Developments}

The notion of functionally compact set we introduced in the present work permits to show that compactly supported GSF are close analogues of classical compactly supported smooth functions. In particular, their functional analytic properties parallel those of the test function space of distribution theory. At the same time, for suitable $K$, the space $\mathcal{G} \mathcal{D}_{K}\left(\widetilde{\mathbb{R}}^{n}\right)$ contains extensions to all $\mathrm{CGF} \mathcal{G}^{s}(\Omega)$ and hence also all Schwartz distributions.

The theory developed here opens the door to addressing several central topics in the theory of nonlinear generalized functions from a new angle. As indicated after Thm. 61, a direct generalization of the integral of compactly supported functions to compactly supported GSF is feasible. An immediate application of this lies in a theory of integration for GSF that we hope will allow to harmonize the Schwartz view of generalized functions as functionals with that prevalent in Colombeau theory of considering generalized functions as pointwise maps. Our approach will take inspiration from Garetto's very fruitful duality theory of locally convex $\widetilde{\mathbb{C}}$-modules $[12,15,13]$.

A further natural development of the present article goes in the direction of a generalization to suitable types of asymptotic gauges (see [20, 19]) and hence to the full and the diffeomorphism invariant Colombeau algebras.

Moreover, one can ask whether $\widetilde{\mathbb{R}}$-valued generalized norms in $\mathcal{G D}_{K}(U)$ permit to generalize results from classical analysis, like a Picard-Lindelöf theorem for ODE with a GSF right hand side, or a Hahn-Banach theorem for functionals $I: \mathcal{G} \mathcal{D}_{K}(U) \longrightarrow \widetilde{\mathbb{R}}$ defined by diffeologically smooth functionals (see [21]) of the type $I_{\varepsilon}: \mathcal{D}_{K_{\varepsilon}}\left(U_{\varepsilon}\right) \longrightarrow \mathbb{R}$, analogously to the way a GSF is defined by a net of smooth functions. 
Acknowledgment: We would like to thank H. Vernaeve for helpful discussions and the anonymous referee for several suggestions that have led to considerable improvements in Sec. 3.

\section{REFERENCES}

[1] Aragona, J., Colombeau, J.F., Juriaans, S.O., Locally convex topological algebras of generalized functions: compactness and nuclearity in a nonlinear context, Trans. Amer. Math. Soc., to appear.

[2] Aragona, J., Fernandez, R., Juriaans, S. O., A Discontinuous Colombeau Differential Calculus. Monatsh. Math. 144 (2005), 13-29.

[3] Aragona, J., Fernandez, R., Juriaans, S.O., Natural topologies on Colombeau algebras, Topol. Methods Nonlinear Anal. 34 (2009), no. 1, 161-180.

[4] Aragona, J., Juriaans, S. O., Some structural properties of the topological ring of Colombeau's generalized numbers, Comm. Algebra 29 (2001), no. 5, 2201-2230.

[5] Aragona, J., Juriaans, S.O., Oliveira, O.R.B., Scarpalézos, D., Algebraic and geometric theory of the topological ring of Colombeau generalized functions, Proc. Edinb. Math. Soc. (2) 51 (2008), no. 3, 545564

[6] Aragona, J., Fernandez, R., Juriaans, S.O., Oberguggenberger, M., Differential calculus and integration of generalized functions over membranes. Monatsh. Math. 166 (2012), 1-18.

[7] Biagioni, H.A., A Nonlinear Theory of Generalized Functions, Lecture Notes in Mathematics 1421, Springer, Berlin, 1990.

[8] Colombeau, J.F., New generalized functions and multiplication of distributions. NorthHolland, Amsterdam, 1984

[9] Colombeau, J.F., Elementary introduction to new generalized functions. North-Holland, Amsterdam, 1985.

[10] Delcroix, A., Hasler, M.F., Pilipovic, S., Valmorin, V., Sequence spaces with exponent weights. Realizations of Colombeau type algebras, Dissertationes Mathematicae 447, 1-73 (2007).

[11] Garetto, C., Topological structures in Colombeau algebras: Topological C-modules and duality theory. Acta Appl. Math. 88, no. 1, 81-123 (2005).

[12] Garetto, C., Topological structures in Colombeau algebras: investigation of the duals of $\mathcal{G}_{c}(\Omega), \mathcal{G}(\Omega)$ and $\mathcal{G}_{\mathcal{S}}\left(\mathbb{R}^{n}\right)$, Monatsh. Math. 146 (2005), no. 3, 203-226.

[13] Garetto, C., Fundamental solutions in the Colombeau framework: applications to solvability and regularity theory, Acta. Appl. Math. 102 (2008), 281-318.

[14] Garetto, C., Closed graph and open mapping theorems for topological $\widetilde{\mathbb{C}}$-modules and applications, Math. Nachr. 282 (2009), no. 8, 1159-1188.

[15] Garetto, C., Hörmann, G., On duality theory and pseudodifferential techniques for Colombeau algebras: generalized delta functionals, kernels and wave front sets, Bull. Acad. Serbe Cl. Sci. (2006), 31, 115-136.

[16] Garetto, C., Vernaeve, H., Hilbert $\widetilde{\mathbb{C}}$-modules: structural properties and applications to variational problems, Trans. Amer. Math. Soc. 363 (2011), no. 4, 2047-2090.

[17] Giordano, P., Kunzinger, M., New topologies on Colombeau generalized numbers and the Fermat-Reyes theorem, J. Math. Anal. and Appl. 399 (2013) 229-238.

[18] Giordano, P., Kunzinger, M., Vernaeve, H., Strongly internal sets and generalized smooth functions, J. Math. Anal. Appl. 422 (2015) 56-71. 
[19] Giordano, P., Luperi Baglini, L., Asymptotic gauges: Generalization of Colombeau type algebras, Math. Nachr. 1-28 (2015).

[20] Giordano, P., Nigsch, E., Unifying order structures for Colombeau algebras, Math. Nachr. Volume 288, Issue 11-12, pages 1286-1302, (2015).

[21] Giordano P., Wu E., Categorical frameworks for generalized functions, Arab. J. Math., Volume 4, Issue 4, 301-328, 2015.

[22] Grosser, M., Kunzinger, M., Oberguggenberger, M., Steinbauer, R., Geometric theory of generalized functions, Kluwer, Dordrecht, 2001.

[23] Mayerhofer, E., Spherical completeness of the non-Archimedean ring of Colombeau generalized numbers, Bull. Inst. Math. Acad. Sin. (N.S.) 2 (2007), no. 3, 769-783.

[24] Mayerhofer, E., On Lorentz geometry in algebras of generalized functions, Proc. Roy. Soc. Edinburgh Sect. A 138 (2008), no. 4, 843-871.

[25] Oberguggenberger, M. Multiplication of Distributions and Applications to Partial Differential Equations, volume 259 of Pitman Research Notes in Mathematics. Longman, Harlow, 1992.

[26] Oberguggenberger, M., Kunzinger, M., Characterization of Colombeau generalized functions by their pointvalues, Math. Nachr. 203 (1999), 147-157.

[27] Oberguggenberger, M., Vernaeve, H., Internal sets and internal functions in Colombeau theory, J. Math. Anal. Appl. 341 (2008) 649-659.

[28] Scarpalézos, D., Some remarks on functoriality of Colombeau's construction; topological and microlocal aspects and applications, Int. Transf. Spec. Fct. 1998, Vol. 6, no. 1-4, 295-307.

[29] Scarpalézos, D., Colombeau's generalized functions: topological structures; microlocal properties. A simplified point of view, I. Bull. Cl. Sci. Math. Nat. Sci. Math. no. 25 (2000), 89-114.

[30] Vernaeve, H., Ideals in the ring of Colombeau generalized numbers, Comm. Algebra (2010) 38(6):2199-2228.

[31] Vernaeve, H. Nonstandard principles for generalized functions, J. Math. Anal. Appl., Volume 384 , Issue 2, 2011, 536-548.

University of Vienna, Austria

E-mail address: paolo.giordano@univie.ac.at

University of Vienna, Austria

E-mail address: michael.kunzinger@univie.ac.at 